Algebra univers. 58 (2008) 303-333

0002-5240/08/030303 - 31, published online May 14, 2008

DOI 10.1007/s00012-008-2071-3

(C) Birkhäuser Verlag, Basel, 2008

Algebra Universalis

\title{
Pseudocomplemented semilattices are finite-to-finite relatively universal
}

\author{
M. E. Adams And JÜrg Schmid \\ To Verra Trnková on the occasion of her $70^{\text {th }}$ birthday.
}

\begin{abstract}
It is shown that the category of directed graphs is isomorphic to a subcategory of the variety $\mathbf{S}$ of all pseudocomplemented semilattices which contains all homomorphisms whose images do not lie in the subvariety $\mathbf{B}$ of all Boolean pseudocomplemented semilattices. Moreover, the functor exhibiting the isomorphism may be chosen such that each finite directed graph is assigned a finite pseudocomplemented semilattice. That is to say, it is shown that the variety $\mathbf{S}$ of all pseudocomplemented semilattices is finite-to-finite B-relatively universal.

This illustrates the complexity of the endomorphism monoids of pseudocomplemented semilattices since it follows immediately that, for any monoid $M$, there exists a proper class of non-isomorphic pseudocomplemented semilattices such that, for each member $S$, the endomorphisms of $S$ which do not have an image contained in the skeleton of $S$ form a submonoid of the endomorphism monoid of $S$ which is isomorphic to $M$.
\end{abstract}

\section{Introduction}

For a class $\mathbf{K}$ of algebras of similar type, let $\mathbf{H}(\mathbf{K}), \mathbf{S}(\mathbf{K})$, and $\mathbf{P}(\mathbf{K})$ respectively denote the classes of all homomorphic images, subalgebras, and products of algebras in $\mathbf{K}$. A class $\mathbf{K}$ is a variety provided $\mathbf{K}=\mathbf{H S P}(\mathbf{K})$, which, by a classical result of Birkhoff [5], is equivalent to being an equational class.

A pseudocomplemented semilattice $(S ; \wedge, *, 0,1)$ is an algebra where $(S ; \wedge)$ is a semilattice with a least element 0 , a greatest element 1 , and a unary operation $*$ such that, for all $s, t \in S, s \wedge t=0$ if and only if $t \leq s^{*}$. The class of pseudocomplemented semilattices is a variety, see Frink [9]. Further, as established by Jones [14] (see also Sankappanavar [26]), the lattice of all subvarieties of pseudocomplemented semilattices ordered by inclusion is a 3-element chain consisting of the trivial variety $\mathbf{T}$ of all 1-element algebras, the variety $\mathbf{B}$ (determined by the identity $x=x^{* *}$ ) of Boolean pseudocomplemented semilattices (where $\left.x \vee y=\left(x^{*} \wedge y^{*}\right)^{*}\right)$, and the variety $\mathbf{S}$ of all pseudocomplemented semilattices.

Presented by J. Adámek.

Received June 17, 2006; accepted in final form May 8, 2007.

2000 Mathematics Subject Classification: Primary 06A12; Secondary 08A35,18B15.

Key words and phrases: pseudocomplemented semilattice, finite-to-finite universal. 
A variety $\mathbf{V}$ of algebras is universal if every category of algebras of finite type (or, equivalently, as shown by Pultr [23], Hedrlín and Pultr [13], and Vopěnka, Hedrlín, and Pultr [31], the category $\mathbf{G}$ of all connected directed graphs together with all compatible mappings) is isomorphic to a full subcategory of $\mathbf{V}$. If an embedding of $\mathbf{G}$ may be effected by a functor $\boldsymbol{\Phi}: \mathbf{G} \longrightarrow \mathbf{V}$ which assigns a finite algebra to each finite graph, then $\mathbf{V}$ is said to be finite-to-finite universal. A number of examples, as well as properties, of universal varieties are already known (see, for example, Pultr and Trnková [24].) In particular, if $\mathbf{V}$ is universal, then, for every monoid $M$, there exists a proper class of non-isomorphic algebras belonging to $\mathbf{V}$ each of which has an endomorphism monoid isomorphic to $M$. If $\mathbf{V}$ is finite-to-finite universal, then, in addition, for a finite monoid $M$, there exist infinitely many non-isomorphic finite algebras in $\mathbf{V}$ with the preceding property.

The variety $\mathbf{S}$ of all pseudocomplemented semilattices is not universal since, for any pseudocomplemented semilattice $S$, the mapping $\gamma_{S}: S \longrightarrow S$ given by $\gamma_{S}(x)=x^{* *}$ is an endomorphism (referred to as the Glivenko endomorphism) onto the skeleton $S^{*}$ of $S$, where $S^{*}=\left\{x^{*}: x \in S\right\}$ is Boolean and belongs to the subvariety B. In particular, if $S$ is not Boolean, then it has a non-trivial endomorphism onto its skeleton $S^{*}$. Furthermore, the endomorphism monoid of $S$ then has at least as many endomorphisms as the skeleton (which is non-trivial whenever the Boolean skeleton is). It follows that every non-trivial pseudocomplemented semilattice has a non-trivial endomorphism. Since, by the above remarks, any universal variety contains a proper class of non-isomorphic algebras each of which has a trivial endomorphism monoid, it follows that the variety $\mathbf{S}$ of all pseudocomplemented semilattices is not universal.

On the other hand, as shown in [3], there does exist a proper class of nonisomorphic pseudocomplemented semilattices for each of which the identity is the only endomorphism which does not have an image contained in the respective skeleton (although, there is no bound on the cardinalities of the skeletons of the pseudocomplemented semilattices in this class). The situation is reminiscent of that for the variety of idempotent semigroups.

Universal varieties of semigroups have been completely characterised by Koubek and Sichler [18]. However, since any non-trivial idempotent semigroup has at least as many non-trivial endomorphism as there are elements in the semigroup, arguing as above, the variety of idempotent semigroups is not universal. This led Demlová and Koubek to introduce a notion of relatively universal. A variety $\mathbf{V}$ is relatively universal to a subvariety $\mathbf{W}$ (or, briefly, $\mathbf{W}$-relatively universal), providing $\mathbf{G}$ is isomorphic to a subcategory of $\mathbf{V}$ whose morphisms consist of all those homomorphisms whose images does not lie in the variety $\mathbf{W}$. In the course of a remarkable series of papers [6], [7], and [8], Demlová and Koubek completely determine which 
varieties of idempotent semigroups are relatively universal. (We remark that precursors to Demlová and Koubek's notion of W-relatively universal date back as far as Sichler [30].)

Our principal result is the following, which, since Boolean algebras with isomorphic endomorphism monoids are isomorphic (Magill [20], Maxson [21], and Schein $[27])$, is sharp.

Theorem 1.1. The variety $\mathbf{S}$ of all pseudocomplemented semilattices is finite-tofinite $\mathbf{B}$-relatively universal, where $\mathbf{B}$ is the subvariety of all Boolean pseudocomplemented semilattices.

An immediate consequence of Theorem 1.1 is a strengthening of the aforementioned result from [3], namely, for any monoid $M$, there exists a proper class of non-isomorphic pseudocomplemented semilattices such that, for each member $S$, the endomorphisms of $S$ which do not have an image contained in $S^{*}$ form a submonoid of the endomorphism monoid of $S$ which is isomorphic to $M$. In passing, we mention that Theorem 1.1 was first conjectured to hold at the time of [3], but we were unable to prove it.

Although the primary objective here is a better understanding of pseudocomplemented semilattices and, in particular, of their endomorphisms, we mention a topical related notion.

A class $\mathbf{K}$ of algebras of similar type is a quasivariety provided $\mathbf{K}=\mathbf{I S P P}_{\mathbf{u}}(\mathbf{K})$, where $\mathbf{I}(\mathbf{K})$ and $\mathbf{P}_{\mathbf{u}}(\mathbf{K})$ respectively denote the classes of all isomorphic images and ultraproducts of algebras in $\mathbf{K}$. Every variety is a quasivariety, but not every quasivariety is a variety.

For a quasivariety $\mathbf{Q}$, let $L(\mathbf{Q})$ denote the lattice (ordered by set inclusion) of all quasivarieties contained in Q. As defined by Sapir (see, for example, Gorbunov $[10]$ ), a variety $\mathbf{V}$ is $\mathbf{Q}$-universal providing that, for any quasivariety $\mathbf{Q}$ of finite type, $L(\mathbf{Q})$ is a homomorphic image of a sublattice of $L(\mathbf{V})$. Amongst the most noteworthy properties of a $Q$-universal variety $\mathbf{V}$ are the facts that a free lattice on $\omega$ free generators is embeddable in $L(\mathbf{V})$ (hence, $L(\mathbf{V})$ fails to satisfy any non-trivial lattice identity) and $|L(\mathbf{V})|=2^{\omega}$.

In [1], it was shown that every finite-to-finite universal variety is $Q$-universal. It is not known whether every finite-to-finite $\mathbf{W}$-relatively universal variety $\mathbf{V}$ is $Q$-universal (see, for example, Koubek and Sichler [19]). It is in this context that we note Theorem 1.1 shows $\mathbf{S}$ is finite-to-finite $\mathbf{B}$-relatively universal whilst, in [2], it was shown that $\mathbf{S}$ is $Q$-universal. That is, pseudocomplemented semilattices are an example in support of the existing conjecture that every finite-to-finite $\mathbf{W}$-relatively universal variety $\mathbf{V}$ is $Q$-universal. 
Returning to Theorem 1.1, to begin the proof we need to find a suitable pseudocomplemented semilattice that will act as a basic component in a šip-type construction (see, for example, Mendelsohn [22] for a lucid general discussion of this technique). In [3], for each undirected connected graph, a pseudocomplemented semilattice was constructed. This appeared to be a promising source to find such algebras. Ultimately this proved inadequate for our purposes. Consequently, in $\S 2.1$ we give a new construction which associates a pseudocomplemented semilattice with every finite undirected graph. Then, in $\S 2.2$, we choose one such algebra in particular, to be denoted $M$.

To establish the universality of a variety, instead of all directed graphs it is sufficient to find a full embedding of the category $\mathbf{G}_{\mathbf{c}}$ of all connected directed graphs which are (i) strongly loopless (that is, for vertices $u$ and $v$, it is never the case that both $(u, v)$ and $(v, u)$ are edges), (ii) for every vertex $v$, there are edges $(u, v)$ and $(v, w)$, and (iii) considered as undirected graphs, are triangle-free (that is, they do not contain a subgraph isomorphic to $K_{3}$, the complete graph on 3 vertices). These properties are required for technical reasons in order to simplify the many constructions to follow. With this in mind, for each $G=(V ; E)$ in $\mathbf{G}_{\mathbf{c}}$, we will consider the $\mathbf{S}$-free product $\coprod_{\mathbf{S}}\left(M_{e}: e \in E\right)$ where, for $e \in E, M_{e}$ denotes an isomorphic copy of $M$, a particular pseudocomplemented semilattice to be specified in $\S 2.2$. We then define a suitable congruence $\Theta$ over $\coprod_{\mathbf{S}}\left(M_{e}: e \in E\right)$ to obtain the $G$-reduced free product $S_{G}=\left(\coprod_{\mathbf{S}}\left(M_{e}: e \in E\right)\right) / \Theta$. The desired functor $\boldsymbol{\Phi}$ will be determined by $\boldsymbol{\Phi}(G)=S_{G}$. The precise definition of $\boldsymbol{\Phi}$ will be given in $\S 3$.

We remark that the use of quotients of free products of algebras which, in some sense, forces them to act like graphs is not new. For example, in [11], Grätzer and Sichler consider a suitably defined quotient of the free $(0,1)$-lattice generated by $V$ for every triangle-connected undirected graph $G=(V ; E)$, a particular instance of a so called $\mathcal{C}$-reduced free product.

Thus, having defined the functor $\boldsymbol{\Phi}$ in $\S 3$, two problems lie ahead. One is to unmask enough characteristics of $G$-reduced free products that it is possible to show that, with respect to their homomorphims, they mimic the compatible mappings of the corresponding graphs. The other is to do so without needing to solve the word problem in the process, and thereby avoid all that this would entail. As will be seen, we do so by the skin of our teeth.

It is to these ends that, in $\S 4$, we begin by first finding relevant properties of the skeletons of $G$-reduced free products. Since each of these is a quotient of a free products of Boolean algebras, our approach will be to use Stone's topological duality for Boolean algebras.

In $\S 5$, we return from the topological setting to essentially an algebraic one. Two constructions are presented which will be used in the proof of Theorem 1.1 
as testing pseudocomplemented semilattices, thereby enabling us to side step the need to find a complete solution of the word problem for $G$-reduced free products.

In $\S 6$, with the information gleaned from $\S 4$ and $\S 5$, we proceed to show that, with respect to homomorphisms, $G$-reduced free products mimic their graphs, thereby completing the proof of Theorem 1.1.

Finally, we conclude with a tantalizing problem in $\S 7$.

\section{Preliminaries}

2.1. The basic construction. An undirected graph $G=(V ; E)$ is a set $V$ of vertices together with a set $E$ of edges the members of which are 2-element subsets of $V$.

The immediate goal of this section is to associate a pseudocomplemented semilattice $S(G)$ to each finite undirected graph $G$ with 4 or more elements (that $G$ has 4 or more elements will not actually be needed until Lemma 2.6).

Recall that, for a pseudocomplemented semilattice $S$, the endomorphism $\gamma_{S}: S \longrightarrow S$ given by $\gamma(x)=x^{* *}$ is referred to as the Glivenko endomorphism. The Glivenko congruence $\Gamma_{S}$ is the congruence on $S$ induced by $\gamma_{S}$, that is $\Gamma_{S}=$ $\left\{(x, y) \in S \times S: x^{*}=y^{*}\right\}$. Accordingly, its congruence classes are called Glivenko classes. A Glivenko class is trivial iff it is a singleton. Note that $S / \Gamma_{S}$ is isomorphic to the skeleton $S^{*}=\left\{x \in S: x=x^{* *}\right\}=\left\{x \in S: x=y^{*}\right.$ for some $\left.y \in S\right\}$ of $S$.

For a finite undirected graph $G=(V ; E)$, let $B(G)$ denote the Boolean lattice whose elements are all subsets of $V$ ordered by inclusion. The pseudocomplemented semilattice $S(G)$ to be associated with $G$ will have a copy of $B(G)$ as its skeleton, and prescribed Glivenko classes as follows: The classes of $\emptyset$ and $V$ are trivial, if $|A|=1$ or $A \in E$, its class is the two-element chain, and for all other $A$ its class is a copy of the Boolean lattice of all subsets of $V \backslash A$.

More formally, let $S(G)$ be the set of all pairs $(A, B) \in B(G) \times B(G)$ satisfying one the four following mutually exclusive conditions:

$\begin{array}{ll}\text { (i) } A=B=\emptyset . & \text { (ii) }|A|=1 \text {, and } B \in\{\emptyset, V\} \text {. }\end{array}$

(iii) $|A|=2, A \in E$, and $B \in\{\emptyset, V\}$. (iv) $|A| \geq 2, A \notin E$, and $A \subseteq B$. and let $\leq$ denote the restriction of the order on $B(G) \times B(G)$ to $S(G)$.

Lemma 2.1. $(S(G) ; \wedge)$ is a semilattice such that, for $(A, B),(C, D) \in S(G)$,

$$
(A, B) \wedge(C, D)= \begin{cases}(A \cap C, B \cap D) & \text { if }|A \cap C| \geq 2 \text { and } A \cap C \notin E, \\ (A \cap C, V) & \text { if } A \cap C \in E \text { and } B=D=V, \\ (A \cap C, \emptyset) & \text { if } A \cap C \in E \text { and } B \cap D \subset V, \\ (A \cap C, V) & \text { if } A \cap C=\{x\} \text { and } B=D=V, \\ (A \cap C, \emptyset) & \text { if } A \cap C=\{x\} \text { and } B \cap D \subset V, \\ (\emptyset, \emptyset) & \text { if } A \cap C=\emptyset \text {. }\end{cases}
$$


Proof. Since $(B(G) ; \subseteq)$ is a poset, so too is $(S(G) ; \leq)$.

Let $(A, B),(C, D) \in S(G)$. Since $(\emptyset, \emptyset) \in S(G)$, it is always the case that a lower bound for $(A, B)$ and $(C, D)$ exists. Suppose that $(R, S) \in S(G)$ is a lower bound of $(A, B)$ and $(C, D)$. In particular, $R \subseteq A \cap C$ and $S \subseteq B \cap D$.

If $|A \cap C| \geq 2$ and $A \cap C \notin E$, then, since $B \cap D \supseteq A \cap C,(A \cap C, B \cap D) \in S(G)$ and $(A, B) \wedge(C, D)=(A \cap C, B \cap D) \in S(G)$.

If $A \cap C=\{x, y\} \in E$, then either $B=D=V$ or $B \cap D \subset V$. If $B=D=V$, then $B \cap D=V,(A \cap C, B \cap D) \in S(G)$, and $(A, B) \wedge(C, D)=(A \cap C, V) \in S(G)$. If $B \cap D \subset V$, then either $R=\{x, y\},\{x\},\{y\}$, or $\emptyset$. In each case, since $(R, S) \in S(G)$, $S=\emptyset$ and, in particular, $(R, S) \leq(\{x, y\}, \emptyset)$. That is, $(A, B) \wedge(C, D)=(A \cap C, \emptyset)$.

Suppose $A \cap C=\{x\}$. If $B=D=V$, it follows that $(A, B) \wedge(C, D)=(A \cap C, V)$. Otherwise, since $B \cap D \subset V$ and $R=\{x\}$ or $\emptyset$, it again follows that $S=\emptyset$, giving $(A, B) \wedge(C, D)=(A \cap C, \emptyset)$.

Finally, if $A \cap C=\emptyset$, then $R=S=\emptyset$ and $(A, B) \wedge(C, D)=(\emptyset, \emptyset)$.

Lemma 2.2. $(S(G) ; \wedge, *,(\emptyset, \emptyset),(V, V))$ is a pseudocomplemented semilattice where $(V, V)^{*}=(\emptyset, \emptyset)$ and, for $(V, V) \neq(A, B) \in S(G),(A, B)^{*}=\left(A^{*}, V\right)$. Hence, the skeleton $S(G)^{*}$ of $S(G)$ is isomorphic to $B(G)$.

Proof. Then, $(V, V)^{*}=(\emptyset, \emptyset)$. If $(V, V) \neq(A, B) \in S(G)$, then it follows that $A \subset V$. Thus, $A^{*} \neq \emptyset$ and, in particular, $\left(A^{*}, V\right) \in S(G)$. By Lemma 2.1, $(A, B) \wedge\left(A^{*}, V\right)=(\emptyset, \emptyset)$. Further, by Lemma 2.1, if $(A, B) \wedge(C, D)=(\emptyset, \emptyset)$, then $A \cap C=\emptyset$. Thus, $C \subseteq A^{*}$ and, hence, $(C, D) \leq\left(A^{*}, V\right)$, as required. Finally, the isomorphism between $S(G)^{*}$ and $B(G)$ is given by sending $(A, V) \in S(G)$ to $A \in B(G)$.

Having established that $S(G)$ is a pseudocomplemented semilattice for any undirected graph $G$, we now consider properties of $S(G)$ and, in particular, endomorphisms of $S(G)$ (see Lemma 2.6).

Lemma 2.3. $S(G)$ is generated by $\{(V \backslash\{x\}, V \backslash\{x\}): x \in V\}$.

Proof. By Lemma 2.2, $(V \backslash\{x\}, V \backslash\{x\})^{* *}=(V \backslash\{x\}, V)$, so $S(G)^{*}$ may be obtained from $\{(V \backslash\{x\}, V \backslash\{x\}): x \in V\}$ by $\wedge$ and $*$. Since $G$ is finite, for $(A, B) \in S(G)$ and $B \subset V,(A, B)=\bigwedge((V \backslash\{x\}, V\}): x \in V \backslash A) \wedge \bigwedge((V \backslash\{x\}, V \backslash\{x\}): x \in V \backslash B)$.

As the following lemma shows, a non-skeletal element is only greater than the zero of the skeleton. This fact will prove crucial in establishing Lemma 2.6 (see Lemma 2.5).

Lemma 2.4. For $(A, B),(C, D) \in S(G)$, if $(A, B) \geq(C, D)^{*} \neq(\emptyset, \emptyset)$, then $(A, B) \in S(G)^{*}$. 
Proof. By Lemma 2.2, $(C, D) \neq(V, V), C \subset V$, and $(C, D)^{*}=\left(C^{*}, V\right)$. Thus, $\emptyset \neq C^{*} \subseteq A$ and $V \subseteq B$. Either $A=V$ and $(A, B)=(V, V)$ or else $A \subset V$ and $(A, B)^{* *}=\left(A^{*}, V\right)^{*}=\left(A^{* *}, V\right)=(A, B)$. Either way, $(A, B) \in S(G)^{*}$.

Although Lemma 2.4 need not be true for all quotients of $S(G)$, as the following shows, when it holds we can sometimes conclude that the naturally induced congruence associated with the quotient contains the Glivenko congruence.

Lemma 2.5. Let $\Theta$ be a congruence on $(S(G) ; \wedge, *,(\emptyset, \emptyset),(V, V))$. If, for some $x \in V,(V \backslash\{x\}, V \backslash\{x\}) \equiv(V \backslash\{x\}, V)(\Theta)$, then $\Theta \supseteq \Gamma_{S(G)}$ or, for some $(A, B),(C, D) \in S(G),(A, B) \not \equiv(A, V)(\Theta)$ and $[(A, B)]_{\Theta} \geq\left[(C, D)^{*}\right]_{\Theta} \neq[(\emptyset, \emptyset)]_{\Theta}$.

Proof. Suppose, for some $x \in V,(V \backslash\{x\}, V \backslash\{x\}) \equiv(V \backslash\{x\}, V)(\Theta)$ and that $\Theta \nsupseteq \Gamma_{S(G)}$, that is, for some $A, B \subseteq V,(A, B) \not \equiv(A, V)(\Theta)$.

First consider the case that, in addition, $(V \backslash\{x\}, V) \equiv(V, V)(\Theta)$. It follows that $(\{x\}, V)=(V \backslash\{x\}, V)^{*} \equiv(V, V)^{*}=(\emptyset, \emptyset)(\Theta)$ and that, for $y \in V \backslash\{x\}$, $(\{y\}, \emptyset)=(\{y\}, V) \wedge(V \backslash\{x\}, V \backslash\{x\}) \equiv(\{y\}, V) \wedge(V \backslash\{x\}, V)=(\{y\}, V)(\Theta)$. Since $A \subseteq V$ is finite, $A=\left\{x_{0}, \ldots, x_{n-1}\right\}$ for some $n<\omega$. If $\left(\left\{x_{i}\right\}, V\right) \equiv(\emptyset, \emptyset)(\Theta)$ for every $i<n$, then $(A, V)=\bigvee\left(\left(\left\{x_{i}\right\}, V\right): i<n\right) \equiv(\emptyset, \emptyset)(\Theta)$, which is absurd. Thus, for some $i<n,\left(\left\{x_{i}\right\}, V\right) \not \equiv(\emptyset, \emptyset)(\Theta)$. Since $x_{i} \neq x$ and $(A, B) \wedge\left(\left\{x_{i}\right\}, V\right)=$ $\left(\left\{x_{i}\right\}, \emptyset\right)$, setting $(C, D)^{*}=\left(V \backslash\left\{x_{i}\right\}, V\right)^{*}=\left(\left\{x_{i}\right\}, V\right)$ will suffice.

Thus, it remains to consider the case that, for every $y \in V,(V \backslash\{y\}, V) \not \equiv$ $(V, V)(\Theta)$. That is, we now need only consider the case when $\Theta\left|S(G)^{*}=\Delta\right| S(G)^{*}$ where $\Theta\left\lceil S(G)^{*}\right.$ denotes the restriction of $\Theta$ to $S(G)^{*}$. If $(V \backslash\{x\}, V \backslash\{x\}) \equiv$ $(V \backslash\{x\}, V)(\Theta)$, then, once more, for $y \in V \backslash\{x\},(\{y\}, \emptyset)=(\{y\}, V) \wedge(V \backslash$ $\{x\}, V \backslash\{x\}) \equiv(\{y\}, V) \wedge(V \backslash\{x\}, V)=(\{y\}, V)(\Theta)$. If there exists $x \neq y \in A$, then $(C, D)^{*}=(V \backslash\{y\}, V)^{*}=(\{y\}, V)$ will suffice since $(A, B) \wedge(\{y\}, V)=$ $(\{y\}, \emptyset) \equiv(\{y\}, V)(\Theta)$. If there does not exist $x \neq y \in A$, then $A=\{x\}$ and $B=\emptyset$. Choose some $y \neq x$. Then $(\{x, y\}, R) \in S(G)$ for some $R \subset V$. Were it the case that $(\{x, y\}, R) \equiv(\{x, y\}, V)(\Theta)$, then it would follow that $(\{x\}, \emptyset)=$ $(\{x\}, V) \wedge(\{x, y\}, R) \equiv(\{x\}, V) \wedge(\{x, y\}, V)=(\{x\}, V)(\Theta)$, contrary to the hypothesis that $(A, B) \not \equiv(A, V)(\Theta)$. Thus, $(\{x, y\}, R) \not \equiv(\{x, y\}, V)(\Theta)$. In particular, since $(\{y\}, \emptyset) \equiv(\{y\}, V)(\Theta)$, the proof is complete if the given $A$ and $B$ are replaced by $\{x, y\}$ and $R$, respectively, in the preceding argument.

Let $\operatorname{End}(S(G))$ denote the monoid (semigroup with identity) of all endomorphisms of $S(G)$ with composition as multiplication. Let $\varphi \in \operatorname{End}(S(G))$ and let $\Theta$ denote the congruence on $S(G)$ induced by $\varphi$. Also, call a mapping $\psi: G \longrightarrow G$ compatible if $\{\psi(x), \psi(y)\} \in E$ whenever $\{x, y\} \in E)$, and let $\operatorname{Aut}(G)$ be the automorphism group of $G$.

Lemma 2.6. Let $\Theta \nsupseteq \Gamma_{S(G)}$. 
(i) If $(A, B) \in S(G)$ and $\varphi((A, B))=(C, D)$, then $|C|=|A|$, and

(ii) for $x \in V, \varphi(\{x\}, V)=(\{\psi(x)\}, V)$ defines a bijective compatible mapping $\psi: G \longrightarrow G$. Since $V$ is finite, $\psi \in \operatorname{Aut}(G)$.

Proof. If, for some $x \in V, \varphi((V \backslash\{x\}, V))=(V, V)$, then $(V \backslash\{x\}, V \backslash\{x\}) \equiv(V \backslash$ $\{x\}, V)(\Theta)$. By Lemma 2.5, for some $(A, B),(C, D) \in S(G),(A, B) \not \equiv(A, V)(\Theta)$ and $[(A, B)]_{\Theta} \geq\left[(C, D)^{*}\right]_{\Theta} \neq[(\emptyset, \emptyset)]_{\Theta}$. Since $\varphi \in \operatorname{End}(S(G))$ and $[(A, B)]_{\Theta} \notin$ $(S(G) / \Theta)^{*}$, this contradicts Lemma 2.4. We conclude that, for $x \in V, \varphi((V \backslash$ $\{x\}, V)) \neq(V, V)$. In particular, $\varphi \mid S(G)^{*}$ is one-to-one. Since $S(G)$ is finite, it follows that $\varphi \mid S(G)^{*}$ is an automorphism. Hence, for any $(A, V) \in S(G)^{*}$, $\varphi(A, V)=(C, V)$ where $|C|=|A|$, completing the verification of (i).

By (i), for $x \in V, \varphi(\{x\}, V)=(\{\psi(x)\}, V)$ defines a bijection $\psi: V \longrightarrow V$. In particular, for $x, y \in V, \varphi(V \backslash\{x\}, V)=(V \backslash\{\psi(x)\}, V)$ and $\varphi((\{x, y\}, V))=$ $\varphi((\{x\}, V) \vee(\{y\}, V))=(\{\psi(x)\}, V) \vee(\{\psi(y)\}, V)=(\{\psi(x), \psi(y)\}, V)$. Suppose $\{x, y\} \in E$. Since $|V| \geq 4$, it is possible to choose $u, v \in V \backslash\{x, y\}$. Then, by Lemma 2.1, $(\{x, y\}, V) \wedge(V \backslash\{u\}, V \backslash\{u\})=(\{x, y\}, \emptyset)=(\{x, y\}, V) \wedge(V \backslash\{v\}, V \backslash$ $\{v\})$. Suppose $\{\psi(x), \psi(y)\} \notin E$. Then, by Lemma 2.1 again, $\varphi((\{x, y\}, V) \wedge(V \backslash$ $\{u\}, V \backslash\{u\}))=(\{\psi(x), \psi(y)\}, V) \wedge(V \backslash\{\psi(u)\}, V \backslash\{\psi(u)\})=(\{\psi(x), \psi(y)\}, V \backslash$ $\{\psi(u)\})$ and $\varphi((\{x, y\}, V) \wedge(V \backslash\{v\}, V \backslash\{v\}))=(\{\psi(x), \psi(y)\}, V) \wedge(V \backslash\{\psi(v)\}, V \backslash$ $\{\psi(v)\})=(\{\psi(x), \psi(y)\}, V \backslash\{\psi(v)\})$. Since $V \backslash\{\psi(u)\} \neq V \backslash\{\psi(v)\}$, this is impossible. We conclude that, whenever $\{x, y\} \in E$, it follows that $\{\psi(x), \psi(y)\} \in$ $E$, as required.

2.2. A particular instance. We now choose a specific graph $G=(V ; E)$ where $\mathrm{V}=\{\mathrm{a}, \mathrm{b}\} \cup\left\{\mathrm{c}_{i}: 0 \leq i<4\right\}$ and $\mathrm{E}=\left\{\left\{\mathrm{a}, \mathrm{c}_{1}\right\},\left\{\mathrm{b}, \mathrm{c}_{3}\right\},\left\{\mathrm{c}_{0}, \mathrm{c}_{2}\right\}\right\} \cup\left\{\left\{\mathrm{c}_{i}, \mathrm{c}_{i+1}\right\}: 0 \leq\right.$ $i<3\}$ (see Figure 1). In particular, $|\mathrm{V}|=6$, which as required above is $\geq 4$. We distinguish the pair of vertices $a, b \in V$ solely for future reference (see $\S 3$ ). Then, $|\operatorname{Aut}(\mathrm{G})|=1$.

Henceforth, let $M$ denote the pseudocomplemented semilattice $S(\mathrm{G})$ for this particular undirected graph G. It is $M$ that will act as the basic component in the šip-type construction presented here. Observe, again for future reference, that corresponding to $\mathrm{a}$ and $\mathrm{b}$ are $(\{\mathrm{a}\}, \mathrm{V})$ and $(\{\mathrm{b}\}, \mathrm{V})$, respectively, which are atoms of $M^{*}$, and $(\mathrm{V} \backslash\{\mathrm{a}\}, \mathrm{V})$ and $(\mathrm{V} \backslash\{\mathrm{b}\}, \mathrm{V})$, respectively, which are co-atoms of $M^{*}$.

By Lemma 2.6, for $\varphi \in \operatorname{End}(S(G))$, if $\Theta \nsupseteq \Gamma_{S(G)}$, then, for $x \in V, \varphi(\{x\}, V)=$ $(\{\psi(x)\}, V)$ defines $\psi \in \operatorname{Aut}(G)$. For $M$, this implies

Lemma 2.7. For $\varphi \in \operatorname{End}(M)$, if $\Theta \nsupseteq \Gamma_{M}$ where $\Theta$ denotes the congruence induced by $\varphi$, then $\varphi$ is the identity.

Proof. By Lemma 2.6 (i), for $\mathrm{x} \in \mathrm{V}, \varphi(\mathrm{V} \backslash\{\mathrm{x}\}, \mathrm{V})=(\mathrm{V} \backslash\{\mathrm{y}\}, \mathrm{V})$ for some $\mathrm{y} \in \mathrm{V}$. By Lemma 2.5 and Lemma 2.4, $(\mathrm{V} \backslash\{\mathrm{x}\}, \mathrm{V} \backslash\{\mathrm{x}\}) \not \equiv(\mathrm{V} \backslash\{\mathrm{x}\}, \mathrm{V})(\Theta)$ for any $\mathrm{x} \in \mathrm{V}$. 


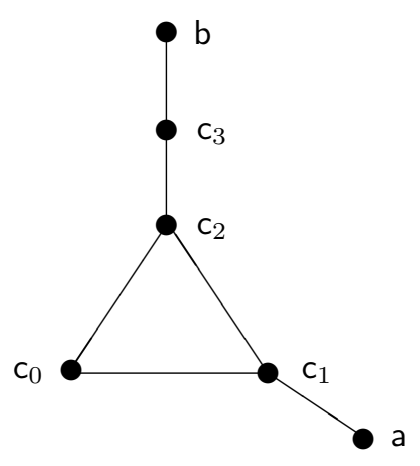

Figure $1 . \mathrm{G}=(\mathrm{V} ; \mathrm{E})$

Thus, by Lemma 2.6, $\varphi((\mathrm{V} \backslash\{\mathrm{x}\}, \mathrm{V} \backslash\{\mathrm{x}\}))=(\mathrm{V} \backslash\{\psi(\mathrm{x})\}, \mathrm{V} \backslash\{\psi(\mathrm{x})\})$ for every $\mathrm{x} \in \mathrm{V}$. Since $M$ is generated by $\{(\mathrm{V} \backslash\{\mathrm{x}\}, \mathrm{V} \backslash\{\mathrm{x}\}): \mathrm{x} \in \mathrm{V}\}$ by Lemma 2.3, it follows from the choice of $\mathrm{G}=(\mathrm{V} ; \mathrm{E})$ that $\varphi$ is the identity.

\section{The functor}

In [12], Hedrlín and Pultr gave a full and faithful embedding from the category of all connected directed graphs to the category of all connected undirected graphs, based on a specific directed graph $G_{0}=\left(V_{0} ; E_{0}\right) \in \mathbf{G}$ with two distinguished vertices $s_{0}$ and $t_{0}$ as diagrammed in Figure 2, respectively on its undirected version $G_{0}^{\prime}=\left(V_{0} ; E_{0}^{\prime}\right)$. They specified a functor $\mathbf{\Psi}$ from $\mathbf{G}$ to the the category of all connected undirected graphs together with all of their compatible mappings as follows: For $G=(V ; E) \in \mathbf{G}$, define an undirected graph $\mathbf{\Psi}(G)$ by taking a copy of $G_{0}^{\prime}$ for every edge in $E$ and, for $e, f \in E$, identifying the element $t_{0}$ of the copy representing $e$ with the element $s_{0}$ of the copy representing $f$ precisely when $e=(u, v)$ and $f=(v, w)$ for vertices $u, v, w \in V$.

If we proceed analogously, but with $G_{0}$ itself instead of $G_{0}^{\prime}$, it is readily seen that we obtain a full and faithful functor $\mathbf{\Psi}: \mathbf{G} \longrightarrow \mathbf{G}_{\mathbf{c}}$, where $\mathbf{G}_{\mathbf{c}}$ denotes the category of all connected directed graphs which are (i) strongly loopless (that is, for vertices $u$ and $v$, it is never the case that both $(u, v)$ and $(v, u)$ are edges), (ii) for every vertex $v$, there are edges $(u, v)$ and $(v, w)$, and (iii) considered as undirected graphs, they are triangle-free (that is, they do not contain a subgraph isomorphic to $K_{3}$, the complete graph on 3 vertices). Thus, in order for us to show that $\mathbf{S}$ is finite-to-finite B-relatively universal, it is sufficient to define a suitable functor $\mathbf{\Phi}: \mathbf{G}_{\mathbf{c}} \longrightarrow \mathbf{S}$. 


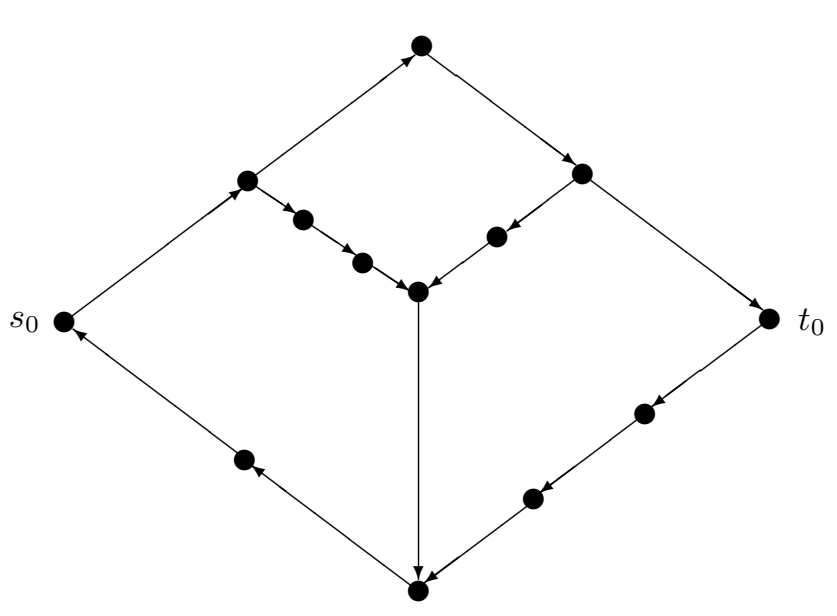

Figure 2. $G_{0}=\left(V_{0} ; E_{0}\right)$

For a variety $\mathbf{V}$ of pseudocomplemented semilattices, let $\left(S_{i}: i \in I\right)$ be a family of pseudocomplemented semilattices such that, for $i \in I, S_{i} \in \mathbf{V}$. A pseudocomplemented semilattice $S \in \mathbf{V}$ is a free $\mathbf{V}$-product of $\left(S_{i}: i \in I\right)$, denoted $\coprod_{\mathbf{V}}\left(S_{i}: i \in I\right)$, providing there is an embedding $\varepsilon_{i}: S_{i} \longrightarrow S$ for every $i \in I$ such that

(i) $S$ is generated by $\bigcup\left(\varepsilon_{i}\left(S_{i}\right): i \in I\right)$ in $\mathbf{V}$, and

(ii) if $T$ is any pseudocomplemented semilattice in $\mathbf{V}$ and, for every $i \in I$, $\varphi_{i}: S_{i} \longrightarrow T$ is a homomorphism, then there exists a homomorphism $\varphi: S \longrightarrow$ $T$ satisfying $\varphi_{i}=\varphi \circ \varepsilon_{i}$ for every $i \in I$.

In [16], Katriňák and Heleyová characterize $\mathbf{S}$-free products. In particular, they show that an $\mathbf{S}$-free product exists provided every component is a singleton or no component is a singleton. We remark that their characterization includes a characterization of free pseudocomplemented semilattices (that is, $\mathbf{S}$-free products of free pseudocomplemented semilattices with 1 free generator, each of which is order isomorphic to a 5 -element non-modular lattice.) Free pseudocomplemented semilattices have also been considered in Balbes [4], Jones [15], and [29]. Although familiarity with [16] will not be required, we will make reference to it.

Let $G=(V ; E)$ be a connected strongly loopless directed graph where, for $v \in V$, there exist $(u, v)$ and $(v, w) \in E$ and, viewed as an undirected graph, no subgraph of it is isomorphic to $K_{3}$ (that is to say, $G \in \mathbf{G}_{\mathbf{c}}$.) For each $e \in E$, let $M_{e}$ denote a copy of the pseudocomplemented semilattice $M$, and $(A, B)_{e}$ denote the copy of $(A, B) \in M$ in $M_{e}$.

Notation: For the rest of this paper $S$ will always denote the free product

$$
S=\coprod_{\mathbf{S}}\left(M_{e}: e \in E\right) .
$$


Let $\Theta_{G}$ be the least congruence on $S$ containing all pairs $\left((\mathrm{V} \backslash\{\mathrm{a}\}, \mathrm{V})_{e},(\mathrm{~V} \backslash\right.$ $\left.\{\mathrm{b}\}, \mathrm{V})_{f}\right)$ and $\left((\mathrm{V} \backslash\{\mathrm{a}\}, \mathrm{V} \backslash\{\mathrm{a}\})_{e},(\mathrm{~V} \backslash\{\mathrm{b}\}, \mathrm{V} \backslash\{\mathrm{b}\})_{f}\right)$ for $e=(u, v)$ and $f=(v, w)$ in $E$.

Let $S_{G}$ be the $G$-reduced free product

$$
S_{G}=\left(\coprod \mathbf{s}\left(M_{e}: e \in E\right)\right) / \Theta_{G}
$$

and set

$$
\Phi(G)=S_{G} .
$$

Note that, since the $\mathbf{S}$-free product exists, $\boldsymbol{\Phi}$ is well-defined on objects. Further, since pseudocomplemented semilattices are locally finite (see Jones [14] and also Sankappanavar [26]), $S_{G}$ is finite for finite $G$. In particular, the functor $\boldsymbol{\Phi}$ is finiteto-finite.

If $h: G \longrightarrow H$ is a compatible mapping between directed graphs $G=(V ; E)$ and $H=(W ; F) \in \mathbf{G}_{\mathbf{c}}$, set

$$
\boldsymbol{\Phi}(h)=\varphi,
$$

where, for $e=(u, v) \in E, \varphi: S_{G} \longrightarrow S_{H}$ is determined by

$$
\varphi\left(\left[(A, B)_{e}\right]_{\Theta_{G}}\right)=\left[(A, B)_{(h(u), h(v))}\right]_{\Theta_{H}}
$$

for $(A, B) \in M$.

Let $\psi: \coprod_{\mathbf{S}}\left(M_{e}: e \in E\right) \longrightarrow \coprod_{\mathbf{S}}\left(M_{f}: f \in F\right)$ be the homomorphism determined by $\psi\left((A, B)_{e}\right)=(A, B)_{(h(u), h(v))}$ for $e=(u, v) \in E$ and let $\theta_{H}: \amalg_{\mathbf{S}}\left(M_{f}: f \in\right.$ $F) \longrightarrow S_{H}$ be the natural homomorphism induced by $\Theta_{H}$. To see that $\varphi$ is a welldefined homomorphism, it is sufficient to show that the congruence $\Theta$ induced on $\coprod_{\mathbf{S}}\left(M_{e}: e \in E\right)$ by $\theta_{H} \circ \psi: \coprod_{\mathbf{S}}\left(M_{e}: e \in E\right) \longrightarrow S_{H}$ contains $\Theta_{G}$. In particular, it is sufficient to show that, whenever $e=(u, v)$ and $f=(v, w) \in E$, each of the pairs $\left((\mathrm{V} \backslash\{\mathrm{a}\}, \mathrm{V})_{e},(\mathrm{~V} \backslash\{\mathrm{b}\}, \mathrm{V})_{f}\right)$ and $\left((\mathrm{V} \backslash\{\mathrm{a}\}, \mathrm{V} \backslash\{\mathrm{a}\})_{e},(\mathrm{~V} \backslash\{\mathrm{b}\}, \mathrm{V} \backslash\{\mathrm{b}\})_{f}\right)$ are elements of $\Theta$. Consider, for example, the pair $\left((\mathrm{V} \backslash\{\mathrm{a}\}, \mathrm{V})_{e},(\mathrm{~V} \backslash\{\mathrm{b}\}, \mathrm{V})_{f}\right)$. By definition, $\psi\left((\mathrm{V} \backslash\{\mathrm{a}\}, \mathrm{V})_{e}\right)=(\mathrm{V} \backslash\{\mathrm{a}\}, \mathrm{V})_{(h(u), h(v))}$ and $\psi\left((\mathrm{V} \backslash\{\mathrm{b}\}, \mathrm{V})_{f}\right)=(\mathrm{V} \backslash\{\mathrm{b}\}, \mathrm{V})_{(h(v), h(w))}$ Since $h: G \longrightarrow H$ is compatible, $(h(u), h(v))$ and $(h(v), h(w)) \in F$. Thus, $\theta_{H}(\mathrm{~V} \backslash$ $\left.\{\mathrm{a}\}, \mathrm{V})_{(h(u), h(v))}\right)=\theta_{H}(\mathrm{~V} \backslash\{\mathrm{b}\}, \mathrm{V})_{(h(v), h(w))}$, as required.

\section{Free products of Boolean algebras}

Let $G=(V ; E)$ be a directed graph in $\mathbf{G}_{\mathbf{c}}$. By Katriňák and Heleyová [16],

$$
S^{*}=\left(\coprod \mathbf{s}\left(M_{e}: e \in E\right)\right)^{*}=\coprod_{\mathbf{B}}\left(M_{e}^{*}: e \in E\right)=: B,
$$

where, by Lemma $2.2, M^{*}=\{(\emptyset, \emptyset)\} \cup\{(A, \mathrm{~V}): \emptyset \neq A \subseteq \mathrm{V}\}$.

Let $\Theta_{G^{*}}$ be the least (Boolean) congruence relation on $B$ containing all pairs $\left((\mathrm{V} \backslash\{\mathrm{a}\}, \mathrm{V})_{e},(\mathrm{~V} \backslash\{\mathrm{b}\}, \mathrm{V})_{f}\right)$ for $e=(u, v)$ and $f=(v, w)$ in $E$ and set 


$$
B_{G}=\left(\coprod_{\mathbf{B}}\left(M_{e}^{*}: e \in E\right)\right) / \Theta_{G^{*}} .
$$

As might be expected, the skeleton $S_{G}^{*}$ of the $G$-reduced free product $S_{G}$ is $B_{G}$, which will be confirmed in due course. Before proceeding to the proof of Theorem 1.1 in $\S 6$, we will establish some properties of $B_{G}$.

Since we are concerned only with Boolean algebras in this section, we can and will use Stone's topological representation for them. Although we will provide some basic facts and terminology, for more background on Boolean algebras and free B-products see, for example, Koppelberg [17].

Associated with each Boolean algebra $(B ; \vee, \wedge, *, 0,1)$ is a compact totally disconnected space $(X ; \rho)$. The set $X$ is the set of prime ideals of $B$. Each element of $B$ is associated with the set of prime ideals to which it does not belong and, as such, the elements of $B$ are recognizable as the clopen subsets of $X$ (which form a basis for $\rho$ ). The Boolean operations join, meet, and complement are realized as set union, intersection, and complement, respectively.

For a family of Boolean algebras $\left(B_{i}: i \in I\right)$, the free $\mathbf{B}$-product $B=\coprod_{\mathbf{B}}\left(B_{i}\right.$ : $i \in I)$ is associated with the cartesian product $P=\prod\left(X_{i}: i \in I\right)$ where the topology is the product topology. In particular, a subset $Y \subseteq P$ is associated with an element of $B$ if and only if $Y$ is a clopen subset of $P$ if and only if $Y$ is a finite union of sets of the type $H_{i_{0}} \times \cdots \times H_{i_{k-1}} \times \prod\left(X_{i}: i \neq i_{0}, \ldots, i_{k-1} \in I\right)$ where $i_{0}, \ldots, i_{k-1}$ is any finite selection of pairwise distinct indices from $I$ and, for each $0 \leq j<k, H_{i_{j}}$ is a clopen subset of $X_{i_{j}}$. For each $j \in I, B$ contains a canonical copy $B_{j}^{\prime}$ of $B_{j}$ as a subalgebra, given explicitly as the collection of all sets $\pi_{j}^{-1}\left(H_{j}\right)$ with $H_{j} \subseteq X_{j}$ clopen (where $\pi_{j}$ is the canonical projection of $P=\prod\left(X_{i}: i \in I\right)$ onto $X_{j}$ ). If $b_{j}$ is an element of $B_{j}$, then we will write $b_{j}^{\prime}$ for the copy of $b_{j}$ in $B_{j}^{\prime}$. Further, if $b_{j}$ is associated with a clopen subset $H_{j}$ of $X_{j}$, then, abusing notation, we will also write $b_{j}^{\prime}$ for $\pi_{j}^{-1}\left(H_{j}\right)$.

Actually, (i) $B$ is generated by $\bigcup\left(B_{i}^{\prime}: i \in I\right)$ (that is, the family $\left(\pi_{i}^{-1}\left(H_{i}\right)\right.$ : $b_{i} \in B_{i}$ for some $\left.i \in I\right)$ is an open subbasis for the product topology on $P$ ) and (ii) whenever $b_{i_{0}}, \ldots, b_{i_{k-1}}$ is any finite collection of non-zero elements of pairwise distinct co-factors $B_{i_{0}}, \ldots, B_{i_{k-1}}$, then the meet of their copies $b_{i_{0}}^{\prime}, \ldots, b_{i_{k-1}}^{\prime}$ in $B$ is non-zero (that is, for pairwise distinct spaces $X_{i_{0}}, \ldots, X_{i_{k-1}}$ and non-empty clopen subsets $H_{i_{j}} \subseteq X_{i_{j}}$, it is always the case that $H_{i_{0}} \times \cdots \times H_{i_{k-1}} \times \prod\left(X_{i}: i \neq\right.$ $i_{1}, \ldots, i_{k} \in I$ ) is non-empty). In fact, properties (i) and (ii) characterize $B$ up to isomorphism. The following special case of (ii) will be of interest. Whenever $i, j \in I$ and $i \neq j$, then $b_{i}^{\prime} \leq b_{j}^{\prime}$ implies $b_{i}^{\prime}=0$ or $b_{j}^{\prime}=1$ for any $b_{i}^{\prime}, b_{j}^{\prime} \in B$.

Let $G=(V ; E)$ be a directed graph which, for the moment, we only assume to be strongly loopless. The topic at hand is the effect of the congruence $\Theta_{G^{*}}$ on $B:=\coprod_{\mathbf{B}}\left(M_{e}^{*}: e \in E\right)$. Given an arrow $e=(u, v) \in E$, let $\sigma e$ and $\tau e$ denote its 
source $u$ and target $v$, respectively. Also, call vertices $u, v \in V$ neighbours provided either $(u, v) \in E$ or $(v, u) \in E$ (but not both, by strong looplessness) are in $E$.

Informally, we will assign labels $a, b$, or $c$ to the arrows of $G$ subject to the following admissibility rule. Whenever, at some vertex, some incoming arrow has label $a$ or some outgoing arrow has label $b$, then at this vertex all incoming arrows must be labelled $a$ and all outgoing arrows must be labelled $b$. Formally, a labelling for $G$ is a map $\ell$ from $E$ into the three-element set $\{a, b, c\}$. A labelling $\ell$ is called $G$-admissible at a vertex $v$ if and only if the following holds: if $\tau e=v=\sigma f$ and $\ell(e)=a$ or $\ell(f)=b$, then $\ell\left(e^{\prime}\right)=a$ for all $e^{\prime}$ with $\tau e^{\prime}=v$ and $\ell\left(f^{\prime}\right)=b$ for all $f^{\prime}$ with $\sigma f^{\prime}=v$. The labelling $\ell$ is called $G$-admissible if and only if it is $G$-admissible at every vertex. Let $\ell_{c}$ be the labelling with constant value $c$ and, for any $v \in V$, let $\ell_{v}$ be the labelling given by $\ell_{v}(e)=a$ if and only if $\tau e=v, \ell_{v}(e)=b$ if and only if $\sigma e=v$, and $\ell_{v}(e)=c$ in all other cases. Then, each of these labellings is $G$-admissible.

Recall that $M=S(\mathrm{G})$ for $\mathrm{G}=(\mathrm{V}, \mathrm{E})$ where $\mathrm{V}=\{\mathrm{a}, \mathrm{b}\} \cup\left\{\mathrm{c}_{k}: 0 \leq k<4\right\}$. In particular, by Lemma $2.2, M^{*}=\{(\emptyset, \emptyset)\} \cup\{(A, \mathrm{~V}): \emptyset \subset A \subseteq \mathrm{V}\}$ ordered point-wise by inclusion. Thus, $M^{*}$ is a finite Boolean algebra with 6 atoms. As such, it has an associated Stone space $(X ; \rho)$ with $|X|=6$ and discrete topology $\rho$. We will identify $X$ with $\vee$ and for each $e \in E, \mathrm{~V}_{e}$ stands for a copy of the discrete space $(\mathrm{V} ; \rho)$ with elements $\left\{\mathrm{a}_{e}, \mathrm{~b}_{e}\right\} \cup\left\{\mathrm{c}_{k, e}: 0 \leq k<4\right\}$. Let $P$ be the product space $P:=\prod\left(\mathrm{V}_{e}: e \in E\right)$.

An explicit description of the Stone space associated with $B_{G^{*}}$ is now obtained as follows. An element $\mathbf{x}=\left(\ldots, x_{e}, \ldots,\right) \in P$ is called $G$-admissible provided it satisfies $\mathrm{x}_{e}=\mathrm{a}_{e}$ if and only if $\mathrm{x}_{f}=\mathrm{b}_{f}$ whenever $\tau e=\sigma f$. It follows that if $\mathbf{x}$ is $G$-admissible, then the labelling $\ell$ of $G$ given by $\ell(e)=a$ whenever $\mathrm{x}_{e}=\mathrm{a}_{e}, \ell(e)=b$ whenever $\mathrm{x}_{e}=\mathrm{b}_{e}$, and $\ell(e)=c$ whenever $\mathrm{x}_{e} \in\left\{\mathrm{c}_{k, e}: 0 \leq k<4\right\}$ is $G$-admissible. Conversely, given a $G$-admissible labelling $\ell$ of $G$, any element $\mathbf{x} \in P$ satisfying $\mathrm{x}_{e}=\mathrm{a}_{e}$ whenever $\ell(e)=a, \mathrm{x}_{e}=\mathrm{b}_{e}$ whenever $\ell(e)=b$, and $\mathrm{x}_{e} \in\left\{\mathrm{c}_{k, e}: 0 \leq k<4\right\}$ whenever $\ell(e)=c$ will be $G$-admissible. In which case, we will say that $\mathbf{x}$ is an instance of $\ell$. Let AD denote the set of all $G$-admissible elements of $P$. Then $B_{G^{*}}$ has as its Stone space the closed subset AD of $P$. The clopen subsets of AD are precisely the sets of the form $H \cap \mathrm{AD}$ for clopen $H \subseteq P$. The canonical projection $\pi_{G}: B \longrightarrow B_{G}$ is given explicitly as the map sending the element of $B$ associated with the clopen set $H \subseteq P$ to the element of $B_{G}$ associated with $H \cap \mathrm{AD}$.

We had agreed to write $b_{e}^{\prime}$ for $\pi_{e}^{-1}\left(H_{e}\right)$ when $b_{e}$ is the element of $M_{e}^{*}$ that is associated with $H_{e} \subseteq \mathrm{V}_{e}$. When $b_{e}$ is an atom of $M_{e}^{*}, b_{e}^{\prime}=\pi_{e}^{-1}\left(\left\{\mathrm{x}_{e}\right\}\right)$ for some $\mathrm{x}_{e} \in \mathrm{V}_{e}$. In this case, abusing notation, we may write $\mathrm{x}_{e}^{\prime}$ instead of $b_{e}^{\prime}$. Likewise, when $b_{e}$ is a co-atom of $M_{e}^{*}$, then $b_{e}^{\prime}=\pi^{-1}\left(\mathrm{~V}_{e} \backslash\left\{\mathrm{x}_{e}\right\}\right)$ for some $\mathrm{x}_{e} \in \mathrm{V}_{e}$. Further abusing notation, we may write $\left(\mathrm{x}_{e}^{*}\right)^{\prime}$ instead of $b_{e}^{\prime}$ in this case. 
In order to make the presentation more readable, we will use the following abbreviations. For $\mathrm{x}_{e} \in \mathrm{V}_{e}$ and $\mathrm{y}_{f} \in \mathrm{V}_{f}$, write $\mathrm{x}_{e} \perp \mathrm{y}_{f}$ to mean $\pi_{G}\left(\mathrm{x}_{e}^{\prime}\right) \wedge \pi_{G}\left(\mathrm{y}_{f}^{\prime}\right)=0$, and $\mathrm{x}_{e} \equiv \mathrm{y}_{f}$ to mean $\pi_{G}\left(\mathrm{x}_{e}^{\prime}\right)=\pi_{G}\left(\mathrm{y}_{f}^{\prime}\right)$. Throughout, $k$ and $k^{\prime}$ will denote natural numbers between 0 and 3 , if not stated otherwise explicitly.

As the following shows, there are enough admissible labellings with a prescribed value.

Lemma 4.1. For any $e \in E$ and $\mathrm{k} \in\{a, b, c\}$ there is a $G$-admissible labelling $\ell$ of $G$ such that $\ell(e)=\mathrm{k}$.

Proof. If $\mathrm{k}=c$, let $\ell=\ell_{c}$. If $\mathrm{k}=a$, let $\ell=\ell_{\tau e}$, and if $\mathrm{k}=b$, let $\ell=\ell_{\sigma e}$.

This already suffices to see that $\Theta_{G^{*}}$ does not collapse any of the subalgebras $\left(M_{e}^{*}\right)^{\prime}$ of $B$.

Lemma 4.2. The restriction of $\Theta_{G^{*}}$ to any of the subalgebras $\left(M_{e}^{*}\right)^{\prime}$ of $B$ is the identity; in other words, $\pi_{G}\left[\left(M_{e}^{*}\right)^{\prime}\right] \cong\left(M_{e}^{*}\right)^{\prime} \cong M_{e}^{*}$.

Proof. It suffices to show that no atom $\mathrm{x}_{e}^{\prime}$ of $\left(M_{e}^{*}\right)^{\prime}$ is collapsed to 0 by $\Theta_{G^{*}}$. Equivalently, it must be shown that, for $\mathrm{x}_{e} \in \mathrm{V}_{e}, \pi_{e}^{-1}\left(\left\{\mathrm{x}_{e}\right\}\right) \cap \mathrm{AD} \neq \emptyset$. Depending on whether $\mathrm{x}_{e}=\mathrm{a}_{e}, \mathrm{~b}_{e}$, or $\mathrm{c}_{k, e}$, let $\mathrm{k}=a, b$, or $c$, respectively. By Lemma 4.1, there is a $G$-admissible labelling $\ell$ of $G$ such that $\ell(e)=\mathrm{k}$, so there is an instance $\mathbf{y}$ of $\ell$ such that $\mathrm{y}_{e}=\mathrm{x}_{e}$. In particular, $\mathbf{y} \in \pi_{e}^{-1}\left(\left\{\mathrm{x}_{e}\right\}\right) \cap \mathrm{AD}$.

Recall that $b_{e}^{\prime} \leq b_{f}^{\prime}$ implies $b_{e}^{\prime}=0$ or $b_{f}^{\prime}=1$ for any $b_{e}^{\prime}, b_{f}^{\prime} \in B$ whenever $e \neq f$. This is no longer true in $B_{G}$. Indeed, if $\tau e=\sigma f$, there is certainly no $G$-admissible labelling $\ell$ of $G$ such that $\ell(e)=\ell(f)=a$. Thus there is no $G$-admissible $\mathbf{y} \in P$ such that $\mathrm{y}_{e}=\mathrm{a}_{e}$ and $\mathrm{y}_{f}=\mathrm{a}_{f}$. In particular, it follows that $\pi_{i}^{-1}\left(\mathrm{a}_{e}\right) \cap \pi_{j}^{-1}\left(\mathrm{a}_{f}\right) \cap \mathrm{AD}=\emptyset$, in other words, $\pi_{G}\left(\mathrm{y}_{e}^{\prime}\right) \wedge \pi_{G}\left(\mathrm{x}_{f}^{\prime}\right)=0$ in $B_{G}$, that is, $\mathrm{a}_{e} \perp \mathrm{a}_{f}$. But this is equivalent to $0 \neq \pi_{G}\left(\mathrm{a}_{e}^{\prime}\right) \leq \pi_{G}\left(\left(\mathrm{a}_{f}^{*}\right)^{\prime}\right) \neq 1$.

We will need to know exactly when $b_{e}^{\prime} \leq b_{f}^{\prime}$ in $B_{G}$. This happens iff $x_{e}^{\prime} \leq\left(x_{f}^{*}\right)^{\prime}$ for any atom $\mathrm{x}_{e}^{\prime} \in\left(M_{e}^{*}\right)^{\prime}$ with $\mathrm{x}_{e}^{\prime} \leq b_{e}^{\prime}$ and any coatom $\left(\mathrm{x}_{f}^{*}\right)^{\prime} \in\left(M_{f}^{*}\right)^{\prime}$ with $b_{f}^{\prime} \leq\left(\mathrm{x}_{f}^{*}\right)$. But $\mathrm{x}_{e}^{\prime} \leq\left(\mathrm{x}_{f}^{*}\right)^{\prime}$ iff $\mathrm{x}_{e}^{\prime} \wedge \mathrm{x}_{f}^{\prime}=0$, so the problem boils down to determining disjoint $\pi_{G}$-images of atoms coming from different co-factors. Following along the lines of the example in the preceding paragraph, we start by listing obstructions - imposed by our constraints on the edges of $G$ - to the existence of admissible labellings with two prescribed values.

Lemma 4.3. Let $e \neq f$ and $\mathrm{k}, \mathrm{l} \in\{a, b, c\}$. A G-admissible labelling $\ell$ satisfying $\ell(e)=\mathrm{k}$ and $\ell(f)=\mathrm{I}$ fails to exist exactly in the following 5 cases:

(i) $\mathrm{k}=\mathrm{I}=a$ and $\tau e$ and $\tau f$ are neighbours,

(ii) $\mathrm{k}=a, \mathrm{I}=b$ and $\tau e$ and $\sigma f$ are neighbours,

(iii) $\mathrm{k}=\mathrm{I}=b$ and $\sigma e$ and $\sigma f$ are neighbours, 
(iv) $\mathrm{k}=c, \mathrm{I}=a$ and $\tau f \in\{\sigma e, \tau e\}$,

(v) $\mathrm{k}=c, \mathrm{I}=b$ and $\sigma f \in\{\sigma e, \tau e\}$.

Proof. Since the labelling with constant value $c$ is admissible for any $G$, it is clear that at least one of $\mathrm{k}, \mathrm{I}$ must be in $\{a, b\}$, which - taking advantage of symmetries — leads to the five cases listed.

(i) Let $\mathrm{k}=\mathrm{I}=a$. Suppose $\tau e$ and $\tau f$ are neighbours and assume, without loss of generality, that there is an arrow $g$ from $\tau e$ to $\tau f$. Since $\ell(f)=a$, we have $\ell(g)=a$ because $\ell$ is $G$-admissible at $\tau f$. But $\ell(e)=a$ and, since $\ell$ is $G$-admissible at $\tau e$, $\ell(g)=b$, a contradiction.

Conversely, assume $\tau e$ and $\tau f$ are not neighbours. If $\tau e=\tau f, \ell_{\tau e}=\ell_{\tau f}$ is a $G$-admissible labelling satisfying $\ell(e)=\ell(f)=a$. If $\tau e \neq \tau f$, define $\ell$ by $\ell(g)=a$ if and only if $\tau g=\tau e$ or $\tau g=\tau f, \ell(g)=b$ if and only if $\sigma g=\tau e$ or $\sigma g=\tau f$, and $\ell(g)=c$ in all other cases. This is $G$-admissible if $\tau e$ and $\tau f$ do not have a common neighbour $v$. If $v$ is such a common neighbour, checking the 4 possible combinations (of arrow directions in and out of $v$ ) shows that no violation of admissibility at $v$ occurs.

(ii) and (iii) are proved analogously.

(iv) Let $\mathrm{k}=c$ and $\mathrm{I}=a$. In particular, $\ell$ is $G$-admissible at $\tau f$. If $\tau f=\sigma e$, then $\ell(f)=a$ implies $\ell(e)=b$, a contradiction. If $\tau f=\tau e$, then $\ell(f)=a$ implies $\ell(e)=a$, a contradiction.

Conversely, suppose $\tau f$ is different from $\sigma e$ and $\tau e$. Then the labelling $\ell_{\tau f}$ satisfies $\ell_{\tau f}(e)=c$ and $\ell_{\tau_{f}}(f)=a$.

(v) is proved analogously.

In more algebraic terms, Lemma 4.3 reads:

Corollary 4.4. Let $e \neq f$ and $\mathrm{x}_{e} \in \mathrm{V}_{e}, \mathrm{y}_{f} \in \mathrm{V}_{f}$. Then $\mathrm{x}_{e} \perp \mathrm{y}_{f}$ in $B_{G}$ exactly if

(i) $\mathrm{x}_{e}=\mathrm{a}_{e}, \mathrm{y}_{f}=\mathrm{a}_{f}$, and $\tau e$ and $\tau f$ are neighbours, or

(ii) $\mathrm{x}_{e}=\mathrm{a}_{e}, \mathrm{y}_{f}=\mathrm{b}_{f}$, and $\tau e$ and $\sigma f$ are neighbours, or

(iii) $\mathrm{x}_{e}=\mathrm{b}_{e}, \mathrm{y}_{f}=\mathrm{b}_{f}$, and $\sigma e$ and $\sigma f$ are neighbours, or

(iv) $\mathrm{x}_{e}=\mathrm{c}_{k, e}, \mathrm{y}_{f}=\mathrm{a}_{f}$, and $\tau f \in\{\sigma e, \tau e\}$, or

(v) $\mathrm{x}_{e}=\mathrm{c}_{k, e}, \mathrm{y}_{f}=\mathrm{b}_{f}$, and $\sigma f \in\{\sigma e, \tau e\}$.

Moreover, if $\mathrm{c}_{k, e} \perp \mathrm{y}_{f}$ for some $k$ and $\mathrm{y}_{f}=\mathrm{a}_{f}$ or $\mathrm{y}_{f}=\mathrm{b}_{f}$, then $\mathrm{c}_{k, e} \perp \mathrm{y}_{f}$ for all $k$.

Proof. The five numbered statements translate the corresponding ones in Lemma 4.3 , while the last assertion follows from (iv) and (v), respectively, since the conditions given do not depend on the particular choice of $k$.

The two following lemmata show that $\pi_{G}$-images of atoms coming from different co-factors are comparable only if they are equal, and that this only occurs for atoms of type $\pi_{G}\left(\mathrm{a}_{e}^{\prime}\right), \pi_{G}\left(\mathrm{~b}_{f}^{\prime}\right)$ when forced directly by admissibility. 
Lemma 4.5. Let $e \neq f, \mathrm{x}_{e} \in \mathrm{V}_{e}, \mathrm{y}_{f} \in \mathrm{V}_{f}$. Then $\pi_{G}\left(\mathrm{x}_{e}^{\prime}\right)$ is comparable with $\pi_{G}\left(\mathrm{y}_{f}^{\prime}\right)$ in $B_{G}$ iff $\mathrm{x}_{e} \equiv \mathrm{y}_{f}$.

Proof. Assume $\pi_{G}\left(\mathrm{x}_{e}^{\prime}\right) \leq \pi_{G}\left(\mathrm{y}_{f}^{\prime}\right)$. Hence, $\mathrm{x}_{e} \perp \mathrm{z}_{f}$ for all $\mathrm{z}_{f} \in \mathrm{V}_{f} \backslash\left\{\mathrm{y}_{f}\right\}$. In particular,

$$
\mathrm{V}_{f} \backslash\left\{\mathrm{y}_{f}\right\} \text { must include } \mathrm{c}_{k, f} \text { for some } k \text {. }
$$

Since $c_{k, e} \not \perp c_{k^{\prime}, f}$ by Corollary 4.4, this implies $\mathrm{x}_{e} \in\left\{\mathrm{a}_{e}, \mathrm{~b}_{e}\right\}$. On the other hand, by the last statement of Corollary 4.4, $\mathrm{V}_{f} \backslash\left\{\mathrm{y}_{f}\right\}$ must then contain $\mathrm{c}_{k, f}$ for all $k$ and thus $\mathrm{y}_{f} \in\left\{\mathrm{a}_{f}, \mathrm{~b}_{f}\right\}$.

Assume $\mathrm{x}_{e}=\mathrm{a}_{e}$. From ( $\dagger$ ) and Corollary 4.4 (iv) we obtain either $\tau e=\sigma f$ or $\tau e=\tau f$. In the first case, $\tau f$ and $\tau e$ are neighbours and we get $\mathrm{a}_{e} \perp \mathrm{a}_{f}$ by Corollary 4.4 (i), thus $\mathrm{y}_{f}=\mathrm{b}_{f}$ and $\mathrm{a}_{e} \equiv \mathrm{b}_{f}$ by admissibility at $\tau e=\sigma f$. In the second, $\tau f$ and $\sigma f$ are neighbours and we arrive at $\mathrm{a}_{e} \equiv \mathrm{a}_{f}$ in the same way, using Corollary 4.4 (ii).

The same line of reasoning works also for $\mathrm{x}_{e}=\mathrm{b}_{e}$.

In $\S 6$ we will also require the dual version of Lemma 4.5.

Corollary 4.6. Let $e \neq f, \mathrm{x}_{e} \in \mathrm{V}_{e}, \mathrm{y}_{f} \in \mathrm{V}_{f}$. Then $\pi_{G}\left(\left(\mathrm{~V}_{e} \backslash\left\{\mathrm{x}_{e}\right\}\right)^{\prime}\right)$ is comparable with $\pi_{G}\left(\left(\mathrm{~V}_{f} \backslash\left\{\mathrm{y}_{f}\right\}\right)^{\prime}\right)$ in $B_{G}$ iff $\pi_{G}\left(\left(\mathrm{~V}_{e} \backslash\left\{\mathrm{x}_{e}\right\}\right)^{\prime}\right)=\pi_{G}\left(\left(\mathrm{~V}_{f} \backslash\left\{\mathrm{y}_{f}\right\}\right)^{\prime}\right)$ iff $\mathrm{x}_{e} \equiv \mathrm{y}_{f}$.

Lemma 4.7. For $e \neq f, \mathrm{x}_{e} \in \mathrm{V}_{e}, \mathrm{y}_{f} \in \mathrm{V}_{f}$, we have $\mathrm{x}_{e} \equiv \mathrm{y}_{f}$ iff either $(i) \tau e=\tau f$, $\mathrm{x}_{e}=\mathrm{a}_{e}$, and $\mathrm{y}_{f}=\mathrm{a}_{f}$, or (ii) $\sigma e=\sigma f, \mathrm{x}_{e}=\mathrm{b}_{e}$, and $\mathrm{y}_{f}=\mathrm{b}_{f}$, or (iii) $\tau e=\sigma f$ and $\mathrm{x}_{e}=\mathrm{a}_{e}$, and $\mathrm{y}_{f}=\mathrm{b}_{f}$.

Proof. One direction of each of the equivalences (i)-(iii) is immediate by the definition of admissibility. For the other direction where $\mathrm{x}_{e} \equiv \mathrm{y}_{f}$, it will suffice to show, using Lemma 4.5 , that $\pi_{G}\left(\mathrm{x}_{e}^{\prime}\right) \not \leq \pi_{G}\left(\mathrm{y}_{f}^{\prime}\right)$ with the exception of the three cases listed.

Let $\mathrm{x}_{e}=\mathrm{c}_{k, e}$. Since $\mathrm{c}_{k, e} \not \perp \mathrm{c}_{k^{\prime}, f}$ for all $k^{\prime}$ by Corollary 4.4 , we have $\pi_{G}\left(\mathrm{c}_{k, e}^{\prime}\right) \not \leq$ $\pi_{G}\left(\mathrm{y}_{f}^{\prime}\right)$ for any $\mathrm{y}_{f}$.

Let $\mathrm{x}_{e}=\mathrm{a}_{e}$. Assume $e$ and $f$ have no vertex in common and consider the admissible labelling $\ell_{\tau e}$ with values $\ell_{\tau e}(e)=a$ and $\ell_{\tau e}(f)=c$, and its instances. Again, it follows that $\mathrm{x}_{e} \not \perp \mathrm{c}_{k^{\prime}, f}$ for all $k^{\prime}$, and thus $\pi_{G}\left(\mathrm{a}_{e}^{\prime}\right) \not \leq \pi_{G}\left(\mathrm{y}_{f}^{\prime}\right)$ for any $\mathrm{y}_{f}$. Assume $e$ and $f$ do have a vertex in common. If $\tau e=\tau f$, then $\mathrm{a}_{e} \equiv \mathrm{a}_{f}$; if $\tau e=\sigma f$, then $\mathrm{a}_{e} \equiv \mathrm{b}_{f}$; if $\sigma e \in\{\sigma f, \tau f\}$, consider the admissible labelling $\ell_{\tau e}$ and argue as above.

The case $\mathrm{x}_{e}=\mathrm{b}_{e}$ is handled dually.

The preceding two lemmata combined with the next lemma, will show in Corollary 4.9 that $\pi_{G}$-images of any two elements from different co-factors are comparable only when forced directly by admissibility. 
Lemma 4.8. Assume $e \neq f$ and consider $\pi_{G}\left(h_{f}^{\prime}\right) \in \pi_{G}\left[\left(M_{f}^{*}\right)^{\prime}\right] \cong\left(M_{f}^{*}\right)^{\prime}$ such that $\pi_{G}\left(h_{f}^{\prime}\right)$ is neither an atom nor a coatom of $\pi_{G}\left[\left(M_{f}^{*}\right)^{\prime}\right]$. Then there is no atom $\pi_{G}\left(\mathrm{x}_{e}^{\prime}\right)$ (where $\left.\mathrm{x}_{e} \in \mathrm{V}_{e}\right)$, and coatom $\pi_{G}\left(\left(\mathrm{y}_{e}^{*}\right)^{\prime}\right)$ (where $\left.\mathrm{y}_{e} \in \mathrm{V}_{e}\right)$ of $\pi_{G}\left[\left(M_{e}^{*}\right)^{\prime}\right]$ such that simultaneously $\pi_{G}\left(\mathrm{x}_{e}^{\prime}\right) \leq \pi_{G}\left(h_{f}^{\prime}\right)$ and $\pi_{G}\left(h_{f}^{\prime}\right) \leq \pi_{G}\left(\left(\mathrm{y}_{e}^{*}\right)^{\prime}\right)$.

Proof. Then, $\pi_{G}\left(h_{f}^{\prime}\right)$ is neither an atom nor a coatom of $\pi_{G}\left[\left(M_{f}^{*}\right)^{\prime}\right]$ iff $h_{f}^{\prime}=$ $\pi_{f}^{-1}\left(H_{f}\right), H_{f} \subseteq \mathrm{V}_{f}, 1<\left|H_{f}\right|<5$. Put $H_{f}^{*}:=\mathrm{V}_{f} \backslash H_{f}$. Now

$$
\pi_{G}\left(\mathrm{x}_{e}^{\prime}\right) \leq \pi_{G}\left(h_{f}^{\prime}\right) \text { iff } \mathrm{x}_{e} \perp \mathrm{z}_{f} \text { for all } \mathrm{z}_{f} \in H_{f}^{*},
$$

and analogously

$$
\pi_{G}\left(h_{f}^{\prime}\right) \leq \pi_{G}\left(\left(\mathrm{y}_{e}^{*}\right)^{\prime}\right) \text { iff } \mathrm{y}_{e} \perp \mathrm{z}_{f} \text { for all } \mathrm{z}_{f} \in H_{f} .
$$

It is clear that $\mathrm{x}_{e} \neq \mathrm{y}_{e}$ whenever $(\dagger)$ and $(\dagger \dagger)$ are satisfied simultaneously.

Suppose, contrary to hypothesis, that there is an atom $\pi_{G}\left(\mathrm{x}_{e}^{\prime}\right)$ and a coatom $\pi_{G}\left(\left(\mathrm{y}_{e}^{*}\right)^{\prime}\right)$ of $\pi_{G}\left[\left(M_{e}^{*}\right)^{\prime}\right]$ such that simultaneously $\pi_{G}\left(\mathrm{x}_{e}^{\prime}\right) \leq \pi_{G}\left(h_{f}^{\prime}\right)$ and $\pi_{G}\left(h_{f}^{\prime}\right) \leq$ $\pi_{G}\left(\left(\mathrm{y}_{e}^{*}\right)^{\prime}\right)$.

By Corollary 4.4 (iv) and (v), $\mathrm{z}_{e} \perp \mathrm{c}_{k, f}$ has a (unique) solution iff either $\tau e \in$ $\{\sigma f, \tau f\}$ or $\sigma e \in\{\sigma f, \tau f\}$. Now at least one of $H_{f}$ and $H_{f}^{*}$ must contain some $\mathrm{c}_{k, f}$, so the solvability of $(\dagger)$ and $(\dagger \dagger)$ implies that exactly one of the four possible relative positions of $e$ and $f$ as indicated prevails. Assume $\mathrm{c}_{k, f} \in H_{f}$ for some $k$, and $\tau e=\sigma f$. Then, by Corollary 4.4 again, $\mathrm{a}_{e}$ is the unique candidate for $\mathrm{y}_{e}$ in ( $\left.\dagger \dagger\right)$. Turning to $(\dagger)$, suppose first that $c_{k^{\prime}, f} \in H_{f}^{*}$ for some $k^{\prime} \neq k$. The argument just used gives, again, $\mathrm{a}_{e}$ as the unique candidate for $\mathrm{x}_{e}$, and thus $\mathrm{x}_{e}=\mathrm{y}_{e}$. If $H_{f}^{*}$ does not contain any $\mathrm{c}_{k, f}$, we must have $H_{f}^{*}=\left\{\mathrm{a}_{f}, \mathrm{~b}_{f}\right\}$. But then by Corollary 4.4 (i)(iii), there is no common solution for $\mathrm{x}_{e} \perp \mathrm{a}_{f}$ and $\mathrm{x}_{e} \perp \mathrm{b}_{f}$. The cases $\tau e=\tau f$ and $\sigma e \in\{\sigma f, \tau f\}$ are handled analogously.

Corollary 4.9. Let $e \neq f$ and $D=\left(\pi_{G}\left[\left(M_{e}^{*}\right)^{\prime}\right] \cap \pi_{G}\left[\left(M_{f}^{*}\right)^{\prime}\right]\right) \backslash\{0,1\}$. Then $D \neq \emptyset$ iff either (i) $\tau e=\tau f$ and $D=\left\{\pi_{G}\left(\mathrm{a}_{e}^{\prime}\right)=\pi_{G}\left(\mathrm{a}_{f}^{\prime}\right), \pi_{G}\left(\left(\mathrm{a}_{e}^{*}\right)^{\prime}\right)=\pi_{G}\left(\left(\mathrm{a}_{f}^{*}\right)^{\prime}\right)\right\}$, or (ii) $\sigma e=\sigma f$ and $D=\left\{\pi_{G}\left(\mathrm{~b}_{e}^{\prime}\right)=\pi_{G}\left(\mathrm{~b}_{f}^{\prime}\right), \pi_{G}\left(\left(\mathrm{~b}_{e}^{*}\right)^{\prime}\right)=\pi_{G}\left(\left(\mathrm{~b}_{f}^{*}\right)^{\prime}\right)\right\}$, or (iii) $\tau e=\sigma f$ and $D=\left\{\pi_{G}\left(\mathrm{a}_{e}^{\prime}\right)=\pi_{G}\left(\mathrm{~b}_{f}^{\prime}\right), \pi_{G}\left(\left(\mathrm{a}_{e}^{*}\right)^{\prime}\right)=\pi_{G}\left(\left(\mathrm{~b}_{f}^{*}\right)^{\prime}\right)\right\}$.

Proof. Combine Lemma 4.5, Lemma 4.7, and Lemma 4.8.

The following lemma says that disjointness of $\pi_{G}$-images of atoms coming from different co-factors is rare, and that it is in some sense unique whenever it occurs. It depends heavily on the fact that $G$ is triangle-free, so we will assume that $G$ is strongly loopless and triangle-free from this point on.

Lemma 4.10. Let $f \neq e, \mathrm{x}_{f} \in \mathrm{V}_{f}$ and suppose $\pi_{G}\left(\mathrm{x}_{f}^{\prime}\right) \notin \pi_{G}\left[\left(M_{e}^{*}\right)^{\prime}\right]$. Then there exists at most one $\mathrm{y}_{e} \in \mathrm{V}_{e}$ such that $\mathrm{y}_{e} \perp \mathrm{x}_{f}$. 
Proof. There are five possibilities for the relative positions of $e$ and $f:(1) \sigma e=\sigma f$, (2) $\tau e=\sigma f$, (3) $\sigma e=\tau f$, (4) $\tau e=\tau f$, and (5) $e$ and $f$ have no vertex in common. Then Lemma 4.7 implies for (1) that $\mathrm{b}_{e} \equiv \mathrm{b}_{f}$, for (2) that $\mathrm{a}_{e} \equiv \mathrm{b}_{f}$, for (3) that $\mathrm{b}_{e} \equiv \mathrm{a}_{f}$, and for (4) that $\mathrm{a}_{e} \equiv \mathrm{a}_{f}$.

Case 1: $\mathrm{x}_{f}=\mathrm{a}_{f}$.

- Assume (1).

$-\mathrm{a}_{f} \perp \mathrm{a}_{e}$ iff $\tau e$ and $\tau f$ are neighbours by Corollary 4.4 (i) which violates triangle-free, so this case is not possible.

$-\mathrm{a}_{f} \perp \mathrm{b}_{e}$ is true since $\mathrm{b}_{e} \equiv \mathrm{b}_{f}$.

$-\mathrm{a}_{f} \perp \mathrm{c}_{k, e}$ iff $\tau f \in\{\sigma e, \tau e\}$ by Corollary 4.4 (iv), contradicting $\sigma e=\sigma f$, so this case is not possible.

- Assume (2).

$-\mathrm{a}_{f} \perp \mathrm{a}_{e}$ is true since $\mathrm{a}_{e} \equiv \mathrm{b}_{f}$.

$-\mathrm{a}_{f} \perp \mathrm{b}_{e}$ iff $\sigma e$ and $\tau f$ are neighbours by Corollary 4.4 (ii) which violates triangle-free, so this case is not possible.

$-\mathrm{a}_{f} \perp \mathrm{c}_{k, e}$ iff $\tau f \in\{\sigma e, \tau e\}$ by Corollary 4.4 (iv), contradicting $\sigma e=\sigma f$, so this case is not possible.

- Assume (3). Since $\mathrm{b}_{e} \equiv \mathrm{a}_{f}$, we infer $\pi_{G}\left(\mathrm{a}_{f}^{\prime}\right)=\pi_{G}\left(\mathrm{~b}_{e}^{\prime}\right) \in \pi_{G}\left[\left(M_{e}^{*}\right)^{\prime}\right]$, violating our hypothesis.

- Assume (4). Since $\mathrm{a}_{e} \equiv \mathrm{a}_{f}$, we infer $\pi_{G}\left(\mathrm{a}_{f}^{\prime}\right)=\pi_{G}\left(\mathrm{a}_{e}^{\prime}\right) \in \pi_{G}\left[\left(M_{e}^{*}\right)^{\prime}\right]$, violating our hypothesis.

- Assume (5).

- $\mathrm{a}_{f} \perp \mathrm{a}_{e}$ iff $\tau e$ and $\tau f$ are neighbours by Corollary 4.4 (ii). If $g_{1}$ is an arrow from $\tau e$ to $\tau f$, then $\mathrm{a}_{f} \equiv \mathrm{a}_{g_{1}} \perp \mathrm{a}_{e}$ since $\mathrm{a}_{e} \equiv \mathrm{b}_{g_{1}}$ by Lemma 4.7. If $g_{2}$ is an arrow from $\tau f$ to $\tau e$, then $\mathrm{a}_{f} \equiv \mathrm{b}_{g_{2}} \perp \mathrm{a}_{e}$ since $\mathrm{a}_{e} \equiv \mathrm{a}_{g_{2}}$ by Lemma 4.7. Note that exactly one of $g_{1}$ and $g_{2}$ exists since $G$ is strongly loopless.

$-\mathrm{a}_{f} \perp \mathrm{b}_{e}$ iff $\sigma e$ and $\tau f$ are neighbours by Corollary 4.4 (ii). If $g_{1}$ is an arrow from $\sigma e$ to $\tau f$, then $\mathrm{a}_{f} \equiv \mathrm{a}_{g_{1}} \perp \mathrm{b}_{e}$ since $\mathrm{b}_{e} \equiv \mathrm{b}_{g_{1}}$ by Lemma 4.7. If $g_{2}$ is an arrow from $\tau f$ to $\sigma e$, then $\mathrm{a}_{f} \equiv \mathrm{b}_{g_{2}} \perp \mathrm{b}_{e}$ since $\mathrm{b}_{e} \equiv \mathrm{a}_{g_{2}}$ by Lemma 4.7.

Note that the two preceding cases exclude one another: $\tau f$ can't be a neighbour of $\sigma e$ and $\tau e$ at the same time as $G$ is triangle-free.

$-\mathrm{a}_{f} \perp \mathrm{c}_{k, e}$ iff $\tau f \in\{\sigma e, \tau e\}$ by Corollary 4.4 (iv), contradicting the fact that $e$ and $f$ have no common vertex.

So $\mathrm{y}_{e} \perp \mathrm{a}_{f}$ has a (unique) solution just for (1): $\mathrm{y}_{e}=\mathrm{b}_{e}$, for (2): $\mathrm{y}_{e}=\mathrm{a}_{e}$ and for (5): $\mathrm{y}_{e}=\mathrm{a}_{e}$ iff $\tau e$ and $\tau f$ are neighbours or $\mathrm{y}_{e}=\mathrm{b}_{e}$ iff $\sigma e$ and $\tau f$ are neighbours, respectively.

Case 2: $\mathrm{x}_{f}=\mathrm{b}_{f}$. 
Arguing as in Case 1, we obtain that $\mathrm{y}_{e} \perp \mathrm{b}_{f}$ has a (unique) solution just for (3): $\mathrm{y}_{e}=\mathrm{b}_{e}$, for (4): $\mathrm{y}_{e}=\mathrm{a}_{e}$ and for (5): $\mathrm{y}_{e}=\mathrm{a}_{e}$ iff $\tau e$ and $\sigma f$ are neighbours or $\mathrm{y}_{e}=\mathrm{b}_{e}$ iff $\sigma e$ and $\sigma f$ are neighbours, respectively.

Case 3: $\mathrm{x}_{f}=\mathrm{c}_{k, f}$.

- Assume (1).

$-\mathrm{c}_{k, f} \perp \mathrm{a}_{e}$ iff $\tau e \in\{\sigma f, \tau f\}$ by Corollary 4.4 (iv), contradicting $\sigma e=\sigma f$, so this case is not possible.

- $\mathrm{c}_{k, f} \perp \mathrm{b}_{e}$ is true by Corollary $4.4(\mathrm{v})$ since $\sigma e \in\{\sigma f, \tau f\}$.

$-\mathrm{c}_{k, f} \perp \mathrm{c}_{k^{\prime}, e}$ is not possible by Corollary 4.4.

- Assume (2).

$-\mathrm{c}_{k, f} \perp \mathrm{a}_{e}$ is true by Corollary 4.4 (iv) since $\tau e \in\{\sigma f, \tau f\}$.

$-\mathrm{c}_{k, f} \perp \mathrm{b}_{e}$ iff $\sigma e \in\{\sigma f, \tau f\}$ by Corollary $4.4(\mathrm{v})$, contradicting $\tau e=\sigma f$, so this case is not possible.

$-\mathrm{c}_{k, f} \perp \mathrm{c}_{k^{\prime}, e}$ is not possible by Corollary 4.4.

- Assume (3).

$-\mathrm{c}_{k, f} \perp \mathrm{a}_{e}$ iff $\tau e \in\{\sigma f, \tau f\}$ by Corollary 4.4 (iv), contradicting $\sigma e=\tau f$, so this case is not possible.

$-\mathrm{c}_{k, f} \perp \mathrm{b}_{e}$ is true by Corollary $4.4(\mathrm{v})$ since $\sigma e \in\{\sigma f, \tau f\}$.

$-c_{k, f} \perp c_{k^{\prime}, e}$ is not possible by Corollary 4.4.

- Assume (4).

$-\mathrm{c}_{k, f} \perp \mathrm{a}_{e}$ is true by Corollary 4.4 (iv) since $\tau e \in\{\sigma f, \tau f\}$.

$-\mathrm{c}_{k, f} \perp \mathrm{b}_{e}$ iff $\sigma e \in\{\sigma f, \tau f\}$ by Corollary $4.4(\mathrm{v})$, contradicting $\tau e=\tau f$, so this case is not possible.

$-c_{k, f} \perp c_{k^{\prime}, e}$ is not possible by Corollary 4.4.

- Assume (5).

$-\mathrm{c}_{k, f} \perp \mathrm{y}_{e}$ is not possible for any $\mathrm{y}_{e} \in \mathrm{V}_{e}$ by Corollary 4.4 (iv) and (v).

So $\mathrm{y}_{e} \perp \mathrm{c}_{k, f}$ has a (unique) solution for (1): $\mathrm{y}_{e}=\mathrm{b}_{e}$, for (2): $\mathrm{y}_{e}=\mathrm{a}_{e}$, for (3): $\mathrm{y}_{e}=\mathrm{b}_{e}$, and for (4): $\mathrm{y}_{e}=\mathrm{a}_{e}$.

Remark: Actually, Lemma 4.10 holds exactly if $G$ is triangle-free. It is not hard to construct, given any three arrows $e, f, g$ forming a triangle, an element $\mathrm{x}_{f} \in \mathrm{V}$ such that $\pi_{G}\left(\mathrm{x}_{f}^{\prime}\right) \notin \pi_{G}\left[\left(M_{e}^{*}\right)^{\prime}\right]$, and two elements $\mathrm{y}_{e} \neq \mathrm{z}_{e} \in \mathrm{V}_{e}$ such that $\mathrm{x}_{f} \perp \mathrm{y}_{e}, \mathrm{z}_{e}$.

Our next concern is sets of atoms coming from different co-factors $\pi_{G}\left[\left(M_{e_{i}}^{*}\right)^{\prime}\right]$ which are pairwise disjoint. It too will depend on the fact that $G$ is triangle-free.

Lemma 4.11. Let $e_{1}, \ldots, e_{n} \in E($ for $n>2)$, and $\mathrm{x}_{e_{i}} \in \mathrm{V}_{e_{i}}$ for $1 \leq i \leq n$. Suppose $\mathrm{x}_{e_{i}} \perp \mathrm{x}_{e_{j}}$ for $1 \leq i<j \leq n$. Then, for some $1 \leq j \leq n$, there exist $\mathrm{y}_{i, e_{j}} \in \mathrm{V}_{e_{j}}$ (for $1 \leq i \leq n)$ such that $\mathrm{x}_{e_{i}} \equiv \mathrm{y}_{i, e_{j}}$ and, in particular, it follows that $n \leq 6$. 
Proof. Let $2<n$. Suppose (with no loss in generality) we find $\mathrm{x}_{e_{i}} \in\left\{\mathrm{a}_{e_{i}}, \mathrm{~b}_{e_{i}}\right\}$ for $i=1,2,3$. Select $\tau e_{i} \in V$ iff $\mathrm{x}_{e_{i}}=\mathrm{a}_{e_{i}}, \sigma e_{i} \in V$ iff $\mathrm{x}_{e_{i}}=\mathrm{b}_{e_{i}}$. The three selected vertices of $G$ are pairwise neighbours by Corollary 4.4, violating triangle-free. So at most two of these $\mathrm{x}_{e_{i}}$ are in $\left\{\mathrm{a}_{e_{i}}, \mathrm{~b}_{e_{i}}\right\}$, and thus (with no loss in generality) $\mathrm{x}_{e_{3}}=\mathrm{c}_{k, e_{3}}$ for some $k$. Suppose $\mathrm{x}_{e_{1}}=\mathrm{a}_{e_{1}}$. From $\mathrm{a}_{e_{1}} \perp \mathrm{c}_{k, e_{3}}$ we infer, using Corollary 4.4 (iv), that $\tau e_{1} \in\left\{\sigma e_{3}, \tau e_{3}\right\}$. But then $\mathrm{a}_{e_{1}} \equiv \mathrm{b}_{e_{3}}$ or $\mathrm{a}_{e_{1}} \equiv \mathrm{a}_{e_{3}}$. The analogous argument based on Corollary 4.4 (v) shows that $\mathrm{x}_{e_{1}}=\mathrm{b}_{e_{1}}$ implies $\mathrm{b}_{e_{1}} \equiv \mathrm{b}_{e_{3}}$ or $\mathrm{b}_{e_{1}} \equiv \mathrm{a}_{e_{3}}$. Finally, $\mathrm{c}_{k, e_{3}} \not \perp \mathrm{c}_{k^{\prime}, e_{i}}$ for any $k^{\prime}$ and $i \neq 3$, so all the atoms $\pi_{G}\left(\mathrm{x}_{e_{i}}^{\prime}\right)$ are in $\pi_{G}\left[\left(M_{e_{3}}^{*}\right)^{\prime}\right]$, and there are at most 6 of them.

Let $1 \leq i<j \leq 6$ and assume that $y_{1}, \ldots, y_{6} \in B_{G}$ satisfy $y_{1} \vee \cdots \vee y_{6}=1$, $y_{i} \wedge y_{j}=0$, and $y_{i} \geq \pi_{G}\left(\mathrm{x}_{e_{i}}^{\prime}\right)$ for $e_{i} \in E$ and $\mathrm{x}_{e_{i}} \in \mathrm{V}_{e_{i}}$. Since $\mathrm{x}_{e_{i}} \perp \mathrm{x}_{e_{j}}$ and

$y_{1} \vee \cdots \vee y_{6}=1$, it follows from Lemma 4.11 that, for some $e \in E, \mathbf{x}_{e_{i}} \equiv z_{i, e}$ for $1 \leq i \leq 6$. However, $y_{i} \wedge \bigvee\left(\pi_{G}\left(\mathrm{z}_{j, e}^{\prime}\right): j \neq i\right) \leq y_{i} \wedge \bigvee\left(y_{j}: j \neq i\right)=0$. In particular, $y_{i} \leq\left(\bigvee\left(\pi_{G}\left(\mathbf{z}_{j, e}^{\prime}\right): j \neq i\right)\right)^{*}=\pi_{G}\left(\mathbf{z}_{i, e}^{\prime}\right)$. That is $y_{i}=\pi_{G}\left(\mathbf{z}_{i, e}^{\prime}\right)$. In $\S 6$, we will need a dual version of this.

Corollary 4.12. Let $1 \leq q, q^{\prime} \leq 6$ and assume $y_{1}, \ldots, y_{6} \in B_{G}$ satisfy $y_{1} \wedge \cdots \wedge y_{6}=$ $0, y_{q} \vee y_{q^{\prime}}=1$ whenever $q \neq q^{\prime}$, and $y_{q} \leq \pi_{G}\left(\left(\mathrm{~V}_{e_{q}} \backslash\left\{\mathrm{x}_{e_{q}}\right\}\right)^{\prime}\right)$ for $e_{q} \in E$ and $\mathrm{x}_{e_{q}} \in \mathrm{V}_{e_{q}}$. Then there exists $e \in E$ such that $\left\{y_{1}, \ldots, y_{6}\right\}=\left\{\pi_{G}\left(\left(\mathrm{~V}_{e} \backslash\left\{\mathrm{x}_{e}\right\}\right)^{\prime}\right): \mathrm{x}_{e} \in \mathrm{V}_{e}\right\}$.

\section{Two constructions}

5.1. Doubling elements of a Boolean lattice. For a subset $X$ of a poset $P$, let $(X]=\{y: y \leq x$ for some $x \in X\}$ and $[X)=\{y: y \geq x$ for some $x \in X\}$. For brevity, whenever $X=\{x\},(x]$ and $[x)$ will be used to denote $(X]$ and $[X)$, respectively. For $X \neq \emptyset, X$ is an order ideal or an order filter providing $X=(X]$ or $[X)$, respectively.

We will now define a particular pseudocomplemented semilattice $B \llbracket F \rrbracket$ for each Boolean lattice $B$ and non-trivial (that is, $\emptyset \subset F \subset B$ ) order filter $F$ defined on it. The skeleton of $B \llbracket F \rrbracket$ will be $B$, and the non-trivial Glivenko classes in $B \llbracket F \rrbracket$ will be 2-element chains containing an element from $(B \backslash F) \backslash\{0\}$.

Thus let

$$
B \llbracket F \rrbracket=(B \times \mathbf{2}) \backslash((F \times\{0\}) \cup\{(0,1)\}),
$$

where 2 denotes the 2 -element chain $\{0,1\}$ with $0<1$. Let $\leq$ denote the restriction of the order on $B \times \mathbf{2}$ to $B \llbracket F \rrbracket$. 
Lemma 5.1. $(B \llbracket F \rrbracket ; \wedge, *,(0,0),(1,1))$ is a pseudocomplemented semilattice such that, for $(a, i),(b, j) \in B \llbracket F \rrbracket$,

$$
(a, i) \wedge(b, j)= \begin{cases}(a \wedge b, i \wedge j) & \text { if } a \wedge b \neq 0, \\ (0,0) & \text { if } a \wedge b=0,\end{cases}
$$

where $(1,1)^{*}=(0,0)$ and, for $(a, i) \neq(1,1),(a, i)^{*}=\left(a^{*}, 1\right)$.

Proof. Since $B \llbracket F \rrbracket$ is a subset of $B \times \mathbf{2},(B \llbracket F \rrbracket ; \leq)$ is a poset.

Since $(0,0) \in B \llbracket F \rrbracket,(a, i)$ and $(b, j)$ always have a lower bound. Say $(c, k)$ is one such. Then, as $c \leq a \wedge b$ and $k \leq i \wedge j,(a \wedge b, i \wedge j)$ will be the greatest lower bound providing it is an element of $B \llbracket F \rrbracket$. If $(a \wedge b, i \wedge j) \notin B \llbracket F \rrbracket$, then either $a \wedge b=0$ and $i \wedge j=1$ or else $a \wedge b \in F \backslash\{0\}$ and $i \wedge j=0$. In the former case, $a \wedge b=0$ and $i \wedge j=1$. Then, since $((0,1)]=\{(0,0),(0,1)\}$ in $B \times \mathbf{2},(a, i) \wedge(b, j)=(0,0)$ in $B \llbracket F \rrbracket$. In the latter case, $a \wedge b \in F \backslash\{0\}$. Then $a, b \in F$ and, in particular, $i=j=1$. Thus, contrary to hypothesis, $i \wedge j=1$, and we conclude that this case does not arise.

Certainly, $(1,1)^{*}=(0,0)$. If $(a, i) \neq(1,1), a<1$. If $(a, i) \wedge(b, j)=(0,0)$, then $a \wedge b=0$ and, in particular, $b \leq a^{*}$. Since $(a, i) \wedge\left(a^{*}, 1\right)=(0,0),(a, i)^{*}=\left(a^{*}, 1\right)$, as required.

Lemma 5.2. For $(a, i),(b, j) \in B \llbracket F \rrbracket$, if $(a, i) \geq(b, j)^{*} \neq(0,0)$, then $(a, i) \in$ $B \llbracket F \rrbracket^{*}$.

Proof. By Lemma 5.1, $(b, j) \neq(1,1), b<1$, and $(b, j)^{*}=\left(b^{*}, 1\right)$. Thus, $0<b^{*} \leq a$ and $1 \leq i$. Either $a=1$ and $(a, i)=(1,1)$ or else $a<1$ and $(a, i)^{* *}=\left(a^{*}, 1\right)^{*}=$ $\left(a^{* *}, 1\right)=(a, 1)=(a, i)$.

We are interested in the special case where $B$ equals $M^{*}$ and $F$ is the nontrivial order filter $\{(\mathrm{V}, \mathrm{V})\}$ on $M^{*}$.

Lemma 5.3. (i) $M^{*} \llbracket\{(\mathrm{V}, \mathrm{V})\} \rrbracket$ is isomorphic to $M_{\mathrm{V}}:=\{(\emptyset, \emptyset),(\mathrm{V}, \mathrm{V})\} \cup\{(A, B) ; \emptyset \subset$ $A \subset \mathrm{V}$ and $B=\emptyset$ or $B=\mathrm{V}\}$, ordered by $\subseteq$ component-wise.

(ii) The function $\varphi_{M}: M \longrightarrow M_{\mathrm{V}}$, given by

$$
\varphi_{M}((A, B))= \begin{cases}(A, \mathrm{~V}) & \text { if } B=\mathrm{V}, \\ (A, \emptyset) & \text { if } B \subset \mathrm{V},\end{cases}
$$

for $(A, B) \in M$, is a homomorphism.

Proof. (i) By Lemma 2.2, $M^{*}=S(\mathrm{G})^{*}$ is isomorphic to $B(\mathrm{G})=\mathcal{P}(\mathrm{V})$, the Boolean lattice of all subsets $\mathrm{V}$, and the order filter $\{(\mathrm{V}, \mathrm{V})\}$ on $M^{*}$ corresponds to the order filter $\{\mathrm{V}\}$ on $\mathcal{P}(\mathrm{V})$ under this isomorphism. Realize $\mathcal{P}(\mathrm{V}) \llbracket\{\mathrm{V}\} \rrbracket$ as defined above but with 2 as $\{\emptyset, \mathrm{V}\}$ ordered by $\emptyset \subset \mathrm{V}$. We obtain an isomorphic copy $M_{\vee}$ of $M^{*} \llbracket\{(\mathrm{V}, \mathrm{V})\} \rrbracket$ as described. 
(ii) Consider $(A, B),(C, D) \in M$. Case 1: $A \cap C=\emptyset$. Then $(A, B) \wedge(C, D)=$ $(\emptyset, \emptyset)$ by Lemma 2.1 and thus $\varphi_{M}((A, B) \wedge(C, D))=(\emptyset, \emptyset)=\varphi_{M}((A, B)) \wedge$ $\varphi_{M}((C, D))$ by Lemma 5.1. Case 2: $A \cap C \neq \emptyset$. Subcase 2.1: $B \cap D=\mathrm{V}$. Then $(A, \mathrm{~V}) \wedge(C, \mathrm{~V})=(A \cap C, \mathrm{~V})$ and thus $\varphi_{M}((A, B) \wedge(C, D))=(A \cap C, \mathrm{~V})=$ $\varphi_{M}((A, \mathrm{~V})) \wedge \varphi_{M}((C, \mathrm{~V}))$. Subcase $2.2: B \cap D \neq \mathrm{V}$. Then, with no loss in generality, $B \neq \mathrm{V}$ and $(A, B) \wedge(C, D)=(A \cap C, Z)$ for some $\emptyset \subseteq Z \subset \mathrm{V}$, which implies $\varphi_{M}((A, B) \wedge(C, D))=(A \cap C, \emptyset)$. But then also $\varphi_{M}((A, B))=(A, \emptyset)$ and thus $\varphi_{M}((A, B)) \wedge \varphi_{M}((C, D))=(A \cap C, \emptyset)$. This shows that $\varphi_{M}$ preserves meets. Preservation of pseudocomplements is immediate from the definitions.

A (closely related) case of interest is the following. Define a nontrivial order ideal $I_{G}$ of $B_{G}$ by putting an element $h \in B_{G}$ into $I_{G}$ iff there are an edge $e \in E$ and a coatom $q$ of $\pi_{G}\left[\left(M_{e}^{*}\right)^{\prime}\right]$ such that $h \leq q$. Consequently, $F_{G}:=B_{G} \backslash I_{G}$ is a nontrivial order filter of $B_{G}$.

Imitating the description of $M^{*} \llbracket\{(\mathrm{V}, \mathrm{V})\} \rrbracket$ given in Lemma 5.3 (i), we can realize 2 as $\{\emptyset, X\}$ this time, ordered by $\emptyset \subset X$ where $X$ is any nonempty set (the choice of $X$ will be one purely of notational convenience, but in most cases it will be chosen to be $\mathrm{V}$ or $\mathrm{V}_{e}$ for some $\left.e \in E\right)$. It is straightforward to see that $B_{G} \llbracket F_{G} \rrbracket$ is isomorphic to $B_{G} \llbracket F_{G} \rrbracket_{X}:=\{(\emptyset, \emptyset)\} \cup\left\{\left(\pi_{G}(H), B\right): \emptyset \neq \pi_{G}(H) \in B_{G}\right.$ and $B=$ $\emptyset$ or $B=X\} \backslash\left\{\left(\pi_{G}(H), \emptyset\right): \pi_{G}(H) \in F_{G}\right\}$, ordered by $\subseteq$ component-wise (where, since it will be only a matter of notational convenience, $B_{G} \llbracket F_{G} \rrbracket_{X} \cong B_{G} \llbracket F_{G} \rrbracket_{\mathrm{V}} \cong$ $B_{G} \llbracket F_{G} \rrbracket \mathrm{v}_{e} \cong B_{G} \llbracket F_{G} \rrbracket$.

Recall that $B_{G}$ contains a canonical copy of $M_{e}^{*}$ as a subalgebra for any $e \in E$ by Lemma 4.2, realized as $\left\{\pi_{G}\left(A^{\prime}\right): \emptyset \subseteq A \subseteq \mathrm{V}_{e}\right\}$ ordered by $\subseteq$. Appealing to Lemma 5.3 (i) again, with $\mathrm{V}_{e}$ in place of $\mathrm{V}$, we see that $M_{e}^{*} \llbracket\left\{\left(\mathrm{V}_{e}, \mathrm{~V}_{e}\right)\right\} \rrbracket$ is isomorphic to $M_{\mathrm{V}_{e}}:=\left\{(\emptyset, \emptyset),\left(\mathrm{V}_{e}, \mathrm{~V}_{e}\right)\right\} \cup\left\{\left(\pi_{G}\left(A^{\prime}\right), B\right): \emptyset \subset A \subset \mathrm{V}_{e}\right.$ and $B=\emptyset$ or $\left.B=\mathrm{V}_{e}\right\}$, ordered by $\subseteq$ component-wise. Observing that $\pi_{G}\left(A^{\prime}\right) \in I_{G}$ whenever $A \subset \mathrm{V}_{e}$, we conclude that $M_{\mathrm{V}_{e}}$ is, in fact, a subalgebra of $B_{G} \llbracket F_{G} \rrbracket \mathrm{v}_{e}$.

Let $\varphi_{e}: M_{e} \longrightarrow B_{G} \llbracket F_{G} \rrbracket \vee$ be the map given by

$$
\varphi_{e}((A, B))= \begin{cases}\left(\pi_{G}\left(A^{\prime}\right), \mathrm{V}\right) & \text { if } B=\mathrm{V}_{e}, \\ \left(\pi_{G}\left(A^{\prime}\right), \emptyset\right) & \text { if } B \subset \mathrm{V}_{e},\end{cases}
$$

By Lemma 5.3 (ii), $\varphi_{e}$ is a homomorphism from $M_{e}$ into $B_{G} \llbracket F_{G} \rrbracket \mathrm{v} \cong B_{G} \llbracket F_{G} \rrbracket$.

Corollary 5.4. There exists a homomorphism $\bar{\varphi}: S=\coprod_{\mathbf{S}}\left(M_{e}: e \in E\right) \longrightarrow$ $B_{G} \llbracket F_{G} \rrbracket$ extending $\varphi_{e}$ for every $e \in E$.

5.2. A test algebra for $G$-reduced free products. Let $F_{G}$ and $I_{G}$ be as above and choose a fixed vertex $f \in E$ for the rest of this paragraph. A subset $Z_{G, f}$ of $I_{G}$ 
is now defined as the set of all $h \in I_{G}$ such that there are an atom $p$ of $\pi_{G}\left[\left(M_{f}^{*}\right)^{\prime}\right]$ and a coatom $q$ of $\pi_{G}\left[\left(M_{f}^{*}\right)^{\prime}\right]$ satisfying $p \leq h \leq q$.

Since $\pi_{G}\left[\left(M_{f}^{*}\right)^{\prime}\right]$ is a finite subalgebra of $B_{G}$, there exists, for any $h \in B_{G}$, a uniquely determined largest element $a \in \pi_{G}\left[\left(M_{f}^{*}\right)^{\prime}\right]$ such that $a \leq h$; we call it the lower cover of $h$ in $\pi_{G}\left[\left(M_{f}^{*}\right)^{\prime}\right]$ and denote it by $\lambda(h)$. Note that $\lambda: B_{G} \longrightarrow$ $\pi_{G}\left[\left(M_{f}^{*}\right)^{\prime}\right]$ is a $\wedge$-retraction of $B_{G}$ onto $\pi_{G}\left[\left(M_{f}^{*}\right)^{\prime}\right]$.

Our goal is to construct a pseudocomplemented semilattice $S_{f}$ with skeleton $B_{G}$ and prescribed Glivenko classes, as follows: Let $h \in B_{G}$. Then its Glivenko class in $S_{f}$ shall be $\{h\}$ if $h=0$ or $h \in F_{G}$, a copy of the Glivenko class of $\lambda(h)$ in $M_{f} \supseteq M_{f}^{*} \cong \pi_{G}\left[\left(M_{f}^{*}\right)^{\prime}\right] \ni \lambda(h)$ if $h \in Z_{G, f}$, and a two-element set if $h \neq 0$ and $h \in I_{G} \backslash Z_{G, f}$. The construction of $S_{f}$ generalizes that of $S(G)$ given in $\S 2$, and is based on the description of $B_{G}$ given in $\S 4$.

Recall that elements $h \in B_{G}$ are represented as sets $\pi_{G}(H)=H \cap \mathrm{AD}$ where $H$ is any clopen subset of $P=\prod\left(\mathrm{V}_{e}: e \in E\right)$. Given such $\pi_{G}(H)$, there is a unique largest subset $A \subseteq \mathrm{V}_{f}$ such that $\pi_{G}\left(A^{\prime}\right) \subseteq \pi_{G}(H)$, to be denoted by $A=\Lambda\left(\pi_{G}(H)\right)$. It is easy to see that whenever $\pi_{G}(H)$ represents $h \in B_{G}$ and $A=$ $\Lambda\left(\pi_{G}(H)\right)$, then the lower cover $\lambda(h)$ of $h$ is represented by $\pi_{G}\left(A^{\prime}\right)$; further, we have $\Lambda\left(\pi_{G}\left(H_{1} \cap H_{2}\right)\right)=\Lambda\left(\pi_{G}\left(H_{1}\right)\right) \cap \Lambda\left(\pi_{G}\left(H_{2}\right)\right)$. Finally, the subset $I_{G}$ of $B_{G}$ defined above is realized as the collection of all $\pi_{G}(H)$ such that $\pi_{G}(H) \subseteq \pi_{G}\left(\left(\mathrm{~V}_{e} \backslash\{\mathrm{y}\}\right)^{\prime}\right)$ for some $e \in E$ and some $\mathrm{y} \in \mathrm{V}_{e}$. Further, the subset $Z_{G, f}$ of $B_{G}$ defined above is realized as the collection of all $\pi_{G}(H)$ such that $\pi_{G}\left(\{\mathrm{x}\}^{\prime}\right) \subseteq \pi_{G}(H) \subseteq \pi_{G}\left(\left(\mathrm{~V}_{f} \backslash\{\mathrm{y}\}\right)^{\prime}\right)$ for some $\mathrm{x}, \mathrm{y} \in \mathrm{V}_{f}$. In particular, $\Lambda\left(\pi_{G}(H)\right) \neq \emptyset$ whenever $\pi_{G}(H) \in Z_{G, f}$.

The pseudocomplemented semilattice $S_{f}$ announced above is now defined as a subset of $B_{G} \times \mathcal{P}\left(\mathrm{V}_{f}\right)$, ordered by set inclusion component-wise (where $\mathcal{P}\left(\mathrm{V}_{f}\right)$ is the power set of $\mathrm{V}_{f}$ ), as follows.

Definition 5.5. A pair $\left(\pi_{G}(H), B\right) \in B_{G} \times \mathcal{P}\left(\mathrm{V}_{f}\right)$ belongs to $S_{f}$ iff one of the following holds:

(i) $\pi_{G}(H)=\emptyset$ and $B=\emptyset$,

(ii) $\pi_{G}(H) \in F_{G}$ and $B=\mathrm{V}_{f}$,

(iii) $\pi_{G}(H) \in Z_{G, f}$, and $\left(\Lambda\left(\pi_{G}(H)\right), B\right) \in M_{f}$,

(iv) $\emptyset \neq \pi_{G}(H) \in I_{G} \backslash Z_{G, f}$, and $B=\emptyset$ or $B=\mathrm{\vee}_{f}$.

The cases listed are mutually exclusive. Writing $\leq$ for the order relation defined on $S_{f}$, we will show that $\left(S_{f}, \leq\right)$ is a pseudocomplemented semilattice. The following fact, a direct consequence of Lemma 4.10, is crucial.

Lemma 5.6. Let $\pi_{G}\left(H_{1}\right) \in Z_{G, f}, \pi_{G}\left(H_{2}\right) \in I_{G} \backslash Z_{G, f}$ and $\pi_{G}\left(H_{1}\right) \subseteq \pi_{G}\left(H_{2}\right)$. Then $\Lambda \pi_{G}\left(H_{1}\right)=\left\{\mathrm{x}_{f}\right\}$ for some $\pi_{G}\left(\mathrm{x}_{f}^{\prime}\right) \in Z_{G, f}$.

Proof. Let $A:=\Lambda \pi_{G}\left(H_{1}\right)$. Then $A \neq \emptyset$ since $\pi_{G}\left(H_{1}\right) \in Z_{G, f}$. Pick $\mathrm{x}_{f} \in A$. It follows that $\pi_{G}\left(\mathrm{x}_{f}^{\prime}\right) \subseteq \pi_{G}\left(A^{\prime}\right) \subseteq \pi_{G}\left(H_{1}\right)$. Now if $\pi_{G}\left(H_{1}\right) \subseteq \pi_{G}\left(H_{2}\right)$, then $\pi_{G}\left(\mathrm{x}_{f}^{\prime}\right) \subseteq$ 
$\pi_{G}\left(H_{2}\right) \in I_{G} \backslash Z_{G, f}$ implies that $\pi_{G}\left(H_{2}\right) \subseteq \pi_{G}\left(\left(\mathrm{~V}_{e} \backslash\left\{\mathrm{y}_{e}\right\}\right)^{\prime}\right)$ for some $\mathrm{y}_{e} \in \mathrm{V}_{e}$ such that $e \neq f$ and $\mathrm{y}_{e} \not \equiv \mathrm{z}_{f}$ for any $\mathrm{z}_{f} \in \mathrm{V}_{f}$. It follows that $\pi_{G}\left(\mathrm{x}_{f}^{\prime}\right) \subseteq \pi_{G}\left(\left(\mathrm{~V}_{e} \backslash\left\{\mathrm{y}_{e}\right\}\right)^{\prime}\right)$, in other words, $\mathrm{x}_{f} \perp \mathrm{y}_{e}$. But by Lemma 4.10, this equation has at most one solution for $\mathrm{x}_{f}$, given $\mathrm{y}_{e}$ as specified.

Lemma 5.7. $\left(S_{f}, \leq\right)$ is a pseudocomplemented semilattice.

Proof. Fix any pair $\left(\pi_{G}\left(H_{1}\right), B_{1}\right),\left(\pi_{G}\left(H_{2}\right), B_{2}\right)$ of elements of $S_{f}$ and consider an arbitrary element $\left(\pi_{G}(H), B\right) \in S_{f}$ such that $\left(\pi_{G}(H), B\right) \leq\left(\pi_{G}\left(H_{i}\right), B_{i}\right)$ for $i=1,2$. We have to show that there is a unique $\leq$-maximal element in $S_{f}$ with this property, to be denoted it by $\left(\pi_{G}\left(H_{m}\right), B_{m}\right)$.

Define $\pi_{G}\left(H_{0}\right):=\pi_{G}\left(H_{1}\right) \cap \pi_{G}\left(H_{2}\right)\left(=\pi_{G}\left(H_{1} \cap H_{2}\right)\right)$ and $B_{0}:=B_{1} \cap B_{2}$. It is clear that $\pi_{G}(H) \subseteq \pi_{G}\left(H_{0}\right)$ and $B \subseteq B_{0}$. Note that $\left(\pi_{G}\left(H_{0}\right), B_{0}\right)$ is not necessarily a member of $S_{f}$. However, we will show that in all cases, we have either $\left(\pi_{G}\left(H_{m}\right), B_{m}\right)=\left(\pi_{G}\left(H_{0}\right), B_{0}\right)\left(\right.$ with $\left.B_{0} \neq \emptyset\right)$ or $\left(\pi_{G}\left(H_{m}\right), B_{m}\right)=\left(\pi_{G}\left(H_{0}\right), \emptyset\right)$.

We may assume that $\pi_{G}\left(H_{0}\right) \neq \emptyset$ for otherwise $\left(\pi_{G}\left(H_{m}\right), B_{m}\right)$ equals $(\emptyset, \emptyset)$. Observe further that $\left(\pi_{G}(H), \vee_{f}\right) \in S_{f}$ for any $\emptyset \neq \pi_{G}(H) \in B_{G}$. This readily implies that $\left(\pi_{G}\left(H_{m}\right), B_{m}\right)=\left(\pi_{G}\left(H_{0}\right), \mathrm{V}_{f}\right)$ whenever $B_{1}=B_{2}=\mathrm{V}_{f}$, and takes care, in particular, of all cases where $\pi_{G}\left(H_{1}\right), \pi_{G}\left(H_{2}\right)$ both are in $F_{G}$ (by Definition 5.5 (ii)) and where $\emptyset \neq \pi_{G}\left(H_{0}\right) \in F_{G}$ (since then both of $\pi_{G}\left(H_{1}\right), \pi_{G}\left(H_{2}\right)$ must be in $\left.F_{G}=B_{G} \backslash I_{G}\right)$. So we may assume $B_{0} \neq \mathrm{V}_{f}$ and $\emptyset \neq \pi_{G}\left(H_{0}\right) \in I_{G}$ in the sequel. We will determine, given such $\pi_{G}\left(H_{0}\right)$ and $B_{0}$, all elements $\left(\pi_{G}(H), B\right) \in S_{f}$ satisfying $\pi_{G}(H) \subseteq \pi_{G}\left(H_{0}\right)$ and $B \subseteq B_{0}$.

For the purpose of this proof, call a subset of $\mathrm{V}_{f}$ small if it is a singleton or an edge of $\mathrm{G}_{f}=\left(\mathrm{V}_{f}, \mathrm{E}_{f}\right)$. We distinguish three cases:

(1) $\pi_{G}\left(H_{0}\right) \in I_{G} \backslash Z_{G, f}$.

(2) $\pi_{G}\left(H_{0}\right) \in Z_{G, f}$ and $\Lambda \pi_{G}\left(H_{0}\right)$ is small.

(3) $\pi_{G}\left(H_{0}\right) \in Z_{G, f}$ and $\Lambda \pi_{G}\left(H_{0}\right)$ is not small.

Assume (1). If $\pi_{G}(H) \subseteq \pi_{G}\left(H_{0}\right)$ then either (1.1) $\pi_{G}(H) \in I_{G} \backslash Z_{G, f}$ or (1.2) $\pi_{G}(H) \in Z_{G, f}$. If (1.1) holds, then $\left(\pi_{G}(H), B\right) \in S_{f}$ implies $B=\emptyset$ by Definition 5.5 (iv) since $B=\mathrm{V}_{f}$ is ruled out by $B \subseteq B_{0} \neq \mathrm{V}_{f}$. If (1.2) holds, then $\Lambda\left(\pi_{G}(H)\right)$ is small by Lemma 5.6 , which also implies $B=\emptyset$ by Definition 5.5 (iii) and the properties of $M_{f} \cong M$. Now $\left(\pi_{G}\left(H_{0}\right), \emptyset\right)$ itself is in $S_{f}$ by Definition 5.5 (iv), and we conclude that indeed $\left(\pi_{G}\left(H_{m}\right), B_{m}\right)=\left(\pi_{G}\left(H_{0}\right), \emptyset\right)$.

Assume (2). If $\pi_{G}(H) \subseteq \pi_{G}\left(H_{0}\right)$ then either (2.1) $\pi_{G}(H) \in I_{G} \backslash Z_{G, f}$ or (2.2) $\pi_{G}(H) \in Z_{G, f}$. If (2.1) holds, then $B=\emptyset$ as in subcase (1.1). If (2.2) holds, then $\Lambda\left(\pi_{G}(H)\right)$ is small since $\pi_{G}(H) \subseteq \pi_{G}\left(H_{0}\right)$ implies $\Lambda\left(\pi_{G}(H)\right) \subseteq \Lambda\left(\pi_{G}\left(H_{0}\right)\right)$, and so $B=\emptyset$ as in subcase (1.2). Moreover, $\left(\pi_{G}\left(H_{0}\right), \emptyset\right) \in S_{f}$ by Definition 5.5 (iii) and the properties of $M_{f}$, and thus again $\left(\pi_{G}\left(H_{m}\right), B_{m}\right)=\left(\pi_{G}\left(H_{0}\right), \emptyset\right)$. 
Assume (3). Observe first that $\pi_{G}\left(H_{0}\right) \in Z_{G, f}$ and $\Lambda \pi_{G}\left(H_{0}\right)$ not small excludes $\pi_{G}\left(H_{i}\right) \in I_{G} \backslash Z_{G, f}$ by Lemma 5.6 for $i=1,2$. So either $\pi_{G}\left(H_{i}\right) \notin I_{G}$ and $B_{i}=\mathrm{V}_{f}$, or $\pi_{G}\left(H_{i}\right) \in Z_{G, f}$ with $\Lambda\left(\pi_{G}\left(H_{i}\right)\right)$ not small and thus $\Lambda\left(\pi_{G}\left(H_{i}\right)\right) \subseteq B_{i} \subseteq \mathrm{V}_{f}$. We conclude that $\Lambda\left(\pi_{G}\left(H_{0}\right)\right)=\Lambda\left(\pi_{G}\left(H_{1} \cap H_{2}\right)\right)=\Lambda\left(\pi_{G}\left(H_{1}\right)\right) \cap \Lambda\left(\pi_{G}\left(H_{2}\right)\right) \subseteq$ $B_{1} \cap B_{2}=B_{0}$ (and thus $B_{0} \neq \emptyset$ ). But this means that $\left(\pi_{G}\left(H_{0}\right), B_{0}\right) \in S_{f}$ by Definition 5.5 (iii) and the properties of $M_{f}$. Hence $\left(\pi_{G}\left(H_{m}\right), B_{m}\right)=\left(\pi_{G}\left(H_{0}\right), B_{0}\right)$ (with $B_{0} \neq \emptyset$ ).

With that, we have established that $\left(S_{f}, \leq\right)$ is a meet-semilattice with zero $(\emptyset, \emptyset)$. Pseudocomplements are easy: consider $\left(\pi_{G}\left(H_{i}\right), B_{i}\right) \in S_{f}$ for $i=1,2$, and suppose $\left(\pi_{G}\left(H_{1}\right), B_{1}\right) \wedge\left(\pi_{G}\left(H_{2}\right), B_{2}\right)=(\emptyset, \emptyset)$. This implies $\pi_{G}\left(H_{1}\right) \cap \pi_{G}\left(H_{2}\right)=\emptyset$. Let $\pi_{G}(H)$ be the complement of $\pi_{G}\left(H_{1}\right)$ in $B_{G}$; then certainly $\pi_{G}\left(H_{2}\right) \subseteq \pi_{G}(H)$, and $\left(\pi_{G}(H), \vee_{f}\right)$ is the largest element of $S_{f}$ disjoint from $\left(\pi_{G}\left(H_{1}\right), B_{1}\right)$.

Consider $M_{f}$. Then $B_{G}$ contains a canonical copy of $M_{f}^{*}$ as a subalgebra by Lemma 4.2, realized as $\left\{\pi_{G}\left(A^{\prime}\right): \emptyset \subseteq A \subseteq \mathrm{\vee}_{f}\right\}$ (ordered by $\subseteq$ ). Observe that if $A \neq \emptyset, \vee_{f}$, then $\pi_{G}\left(A^{\prime}\right) \in Z_{G, f}$ and $\Lambda\left(\pi_{G}\left(A^{\prime}\right)\right)=A$ by the definition of $\Lambda$. It follows that $\psi_{f}: M_{f} \longrightarrow S_{f}$, defined by

$$
\psi_{f}((A, B))= \begin{cases}(\emptyset, \emptyset) & \text { if } A=\emptyset \\ \left(\pi_{G}\left(A^{\prime}\right), B\right) & \text { otherwise }\end{cases}
$$

is an embedding of $M_{f}$ into $S_{f}$.

Consider $M_{e}, e \neq f$ and the description of $M^{*} \llbracket\{(\mathrm{V}, \mathrm{V})\} \rrbracket$ given in the proof of Lemma 5.3. Using $\mathcal{P}\left(\mathrm{V}_{e}\right)$ as the Boolean lattice and realizing $\mathbf{2}$ as $\left\{\emptyset, \mathrm{V}_{f}\right\}$ (ordered by $\emptyset \subset \mathrm{V}_{f}$ ), we obtain an isomorphic copy of $M_{e}^{*} \llbracket\left\{\left(\mathrm{V}_{e}, \mathrm{~V}_{e}\right)\right\} \rrbracket$ given as $M_{\mathrm{V}_{e}}:=\left\{(\emptyset, \emptyset),\left(\mathrm{V}_{e}, \mathrm{~V}_{f}\right)\right\} \cup\left\{(A, B): \emptyset \subset A \subset \mathrm{V}_{e}\right.$ and $B=\emptyset$ or $\left.B=\mathrm{V}_{f}\right\}$, ordered by $\subseteq$ component-wise.

Again, $B_{G}$ contains a canonical copy of $M_{e}^{*}$ as a subalgebra, realized as $\left\{\pi_{G}\left(A^{\prime}\right)\right.$ : $\left.\emptyset \subseteq A \subseteq \mathrm{V}_{e}\right\}$ (ordered by $\subseteq$ ). Define $\varepsilon_{e}: M_{\mathrm{V}_{e}} \longrightarrow S_{f} \subseteq B_{G} \times \mathcal{P}\left(\mathrm{V}_{f}\right)$ by

$$
\varepsilon_{e}((A, B))= \begin{cases}\left(\pi_{G}\left(A^{\prime}\right), \vee_{f}\right) & \text { if } B=\vee_{e}, \\ \left(\pi_{G}\left(A^{\prime}\right), \emptyset\right) & \text { if } B=\emptyset .\end{cases}
$$

We show that $\varepsilon_{e}$ is an embedding of $M_{\mathrm{V}_{e}}$ into $S_{f}$, provided $\pi_{G}\left[\left(M_{e}^{*}\right)^{\prime}\right] \cap \pi_{G}\left[\left(M_{f}^{*}\right)^{\prime}\right]=$ $\{0,1\}$. Assume not. Then there is $\emptyset \subset A_{e} \subset \mathrm{V}_{e}$ such that $\pi_{G}\left(A_{e}^{\prime}\right) \in \pi_{G}\left[\left(M_{e}^{*}\right)^{\prime}\right] \cap$ $\pi_{G}\left[\left(M_{f}^{*}\right)^{\prime}\right]$. It follows by Corollary 4.9 that $\pi_{G}\left(A_{e}^{\prime}\right)=\pi_{G}\left(A_{f}^{\prime}\right)$ (where $\emptyset \neq A_{f} \neq \mathrm{V}_{f}$ is of the same cardinality as $\left.A_{e}\right)$ is a uniquely determined common atom or coatom of $\pi_{G}\left[\left(M_{e}^{*}\right)^{\prime}\right]$ and $\pi_{G}\left[\left(M_{f}^{*}\right)^{\prime}\right]$, and thus $\pi_{G}\left(A_{f}^{\prime}\right) \in Z_{G, f}$. We need to redefine $\varepsilon_{e}((A, B))$ in case $B=\emptyset$ to account for clause (iii) of Definition 5.5. Recall that $\Lambda\left(\pi_{G}\left(A_{f}^{\prime}\right)\right)=A_{f}$. If $\pi_{G}\left(A_{f}^{\prime}\right)$ is an atom, $\left(A_{f}, C\right) \in M_{f}$ iff $C=\emptyset$ and no modification of $\varepsilon_{e}$ is necessary. So assume $\pi_{G}\left(A_{f}^{\prime}\right)$ is a coatom. To obtain $\left(A_{f}, C\right) \in M_{f}$ with $C \neq \mathrm{V}_{f}$, we must set $\varepsilon_{e}\left(\left(A_{e}, \emptyset\right)\right):=\left(\pi_{G}\left(A_{f}^{\prime}\right), A_{f}\right)=\left(\pi_{G}\left(A_{e}^{\prime}\right), A_{f}\right)$ in this case. 
It remains to show that $\varepsilon_{e}$ so modified preserves meets. Consider $\left(A_{1}, B_{1}\right) \in$ $M \mathrm{~V}_{e}$ such that $\left(A_{1}, B_{1}\right) \neq\left(A_{e}, \emptyset\right)$; the meet of $\left(A_{e}, \emptyset\right)$ and $\left(A_{1}, B_{1}\right)$ in $M_{\mathrm{V}_{e}}$ is $\left(A_{e} \cap A_{1}, \emptyset\right)$. Turning to $\varepsilon_{e}$-images, observe that neither $\pi_{G}\left(A_{1}^{\prime}\right)$ nor $\pi_{G}\left(\left(A_{e} \cap A_{1}\right)^{\prime}\right)$ are in $Z_{G, f}$ by Corollary 4.9. So $\varepsilon_{e}\left(\left(A_{1}, B_{1}\right)\right)$ is either $\left(\pi_{G}\left(A_{1}^{\prime}\right), \mathrm{V}_{f}\right)$ or $\left(\pi_{G}\left(A_{1}^{\prime}\right), \emptyset\right)$, and $\varepsilon_{e}\left(\left(A_{e} \cap A_{1}, \emptyset\right)\right)$ is $\left(\pi_{G}\left(\left(A_{e} \cap A_{1}\right)^{\prime}\right), \emptyset\right)$, while still $\varepsilon_{e}\left(\left(A_{e}, \emptyset\right)\right)=\left(\pi_{G}\left(A_{e}^{\prime}\right), A_{f}\right)$. By the proof of Lemma 5.7, case (1), the meet - taken in $S_{f}-$ of $\left(\pi_{G}\left(A_{e}^{\prime}\right), A_{f}\right)=$ $\left(\pi_{G}\left(A_{f}^{\prime}\right), A_{f}\right)$ with either $\left(\pi_{G}\left(A_{1}^{\prime}\right), \mathrm{V}_{f}\right)$ or $\left(\pi_{G}\left(A_{1}^{\prime}\right), \emptyset\right)$ is indeed $\left(\pi_{G}\left(\left(A_{f} \cap A_{1}\right)^{\prime}\right), \emptyset\right)=$ $\left(\pi_{G}\left(\left(A_{e} \cap A_{1}\right)^{\prime}\right), \emptyset\right)$, as required.

On the other hand, we have the homomorphism $\varphi_{M_{e}}: M_{e} \longrightarrow M_{\mathrm{V}_{e}}$ provided by Lemma 5.3. The composition $\varepsilon_{e} \circ \varphi_{M_{e}}$ thus defines a homomorphism $\psi_{e}: M_{e} \longrightarrow S_{f}$ given explicitly by

$$
\psi_{e}((A, B))=\left\{\begin{array}{cl}
\left(\pi_{G}\left(A^{\prime}\right), \mathrm{V}_{f}\right) & \text { if } B=\mathrm{V}_{e}, \\
\left(\pi_{G}\left(A^{\prime}\right), A_{f}\right) & \text { if } B \subset \mathrm{V}_{e},|A|=5, \text { and } \\
& \pi_{G}\left(A^{\prime}\right)=\pi_{G}\left(A_{f}^{\prime}\right) \in Z_{G, f} \text { for } A_{f} \subset \mathrm{V}_{f}, \\
\left(\pi_{G}\left(A^{\prime}\right), \emptyset\right) & \text { if } B \subset \mathrm{V}_{e}, \text { otherwise }
\end{array}\right.
$$

Corollary 5.8. There exists a homomorphism $\bar{\psi}: S=\coprod_{\mathbf{S}}\left(M_{e}: e \in E\right) \longrightarrow S_{f}$ extending $\psi_{f}$ and $\psi_{e}$ for every $e \in E$ with $e \neq f$.

\section{Proof of Theorem 1.1}

To establish Theorem 1.1, it is sufficient to show that (i) for any directed graphs $G$ and $H \in \mathbf{G}_{\mathbf{c}}, \Phi(h)\left(S_{G}\right) \nsubseteq S_{H}^{*}$ whenever $h: G \longrightarrow H$ is a compatible mapping, and that (ii) there exists a compatible mapping $h: G \longrightarrow H$ such that $\varphi=\Phi(h)$ whenever $\varphi: S_{G} \longrightarrow S_{H}$ is a homomorphism for which $\varphi\left(S_{G}\right) \nsubseteq S_{H}^{*}$.

Let $\bar{\pi}_{G}: S \longrightarrow S_{G}$ be the canonical projection with kernel $\Theta_{G}$. There is an intimate connection between $\bar{\pi}_{G}$ and $\pi_{G}$. The canonical copy of $M_{e}^{*}$ within $S_{G}=$ $S / \Theta_{G}$ is $\left\{\bar{\pi}_{G}\left((A, \mathrm{~V})_{e}\right)\right\}$ for $e \in E$ and $A \subseteq \mathrm{V}$ and the canonical copy of $M_{e}^{*}$ within $B_{G}$, as constructed in $\S 4$, is $\left\{\pi_{G}\left(A^{\prime}\right)\right\}$ for $A \subseteq \mathrm{V}_{e}$. We will freely switch between these representations without explicitly specifying the associated isomorphism.

We start by observing that $\Theta_{G} \subseteq \operatorname{ker} \bar{\varphi}$ and $\Theta_{G} \subseteq \operatorname{ker} \bar{\psi}$ where $\bar{\varphi}: S \longrightarrow B_{G} \llbracket F_{G} \rrbracket$ and $\bar{\psi}: S \longrightarrow S_{f}$ (for any $f \in E$ ) are the homomorphisms given by Corollaries 5.4 and 5.8, respectively. Indeed, recall that $\Theta_{G}$ is the least congruence on $S$ containing all pairs $\left((\mathrm{V} \backslash\{\mathrm{a}\}, \mathrm{V})_{e},(\mathrm{~V} \backslash\{\mathrm{b}\}, \mathrm{V})_{f}\right)$ and $\left((\mathrm{V} \backslash\{\mathrm{a}\}, \mathrm{V} \backslash\{\mathrm{a}\})_{e},(\mathrm{~V} \backslash\{\mathrm{b}\}, \mathrm{V} \backslash\{\mathrm{b}\})_{f}\right)$ for $e=(u, v)$ and $f=(v, w)$ in $E$. But then $\tau e=\sigma f$ and $\pi_{G}\left((\bigvee \backslash\{\mathrm{a}\})_{e}^{\prime}\right)=$ $\pi_{G}\left(\left(\mathrm{~V}_{e} \backslash\left\{\mathrm{a}_{e}\right\}\right)^{\prime}\right)=\pi_{G}\left(\left(\mathrm{~V}_{f} \backslash\left\{\mathrm{b}_{f}\right\}\right)^{\prime}\right)=\pi_{G}\left((\mathrm{~V} \backslash\{\mathrm{b}\})_{f}^{\prime}\right)$. It follows that the partial homomorphisms $\varphi_{e}$ and $\psi_{e}$ used to define $\bar{\varphi}$ and $\bar{\psi}$ take the same values on both components of the pairs generating $\Theta_{G}$ (check the definitions of $\varphi_{e}$ and $\psi_{e}$ ), and so these pairs are in $\operatorname{ker} \bar{\varphi}$ and $\operatorname{ker} \bar{\psi}$, respectively. 
It follows that there exists homomorphisms

$$
\varphi_{G}: S_{G} \longrightarrow B_{G} \llbracket F_{G} \rrbracket \text { and } \psi_{G}: S_{G} \longrightarrow S_{f}
$$

such that $\bar{\varphi}=\varphi_{G} \circ \bar{\pi}_{G}$ and $\bar{\psi}=\psi_{G} \circ \bar{\pi}_{G}$.

There are a number of immediate conclusions that we wish to make from these observations, namely Lemmas 6.1-6.5.

Lemma 6.1. $\bar{\pi}_{G}$ is one-to-one on $M_{e}$ for each $e \in E$.

Proof. Consider some fixed $f \in E$. Then $\bar{\psi}$ extends $\psi_{f}$ (see $\S 5.2$ ) which embeds $M_{f}$ into $S_{f}$. Hence $\psi_{G} \circ \bar{\pi}_{G}$, and with that, $\bar{\pi}_{G}$ must be one-to-one on $M_{f}$.

Lemma 6.2. Let $x \in M_{e}$. Then $\bar{\pi}_{G}(x) \in S_{G}^{*}$ iff $x \in M_{e}^{*}$.

Proof. Consider $x \in M_{e} \backslash M_{e}^{*}$. If $\bar{\pi}_{G}(x) \in S_{G}^{*}$, then $\bar{\pi}_{G}\left(x^{* *}\right)=\bar{\pi}_{G}(x)$. But $x \neq x^{* *} \in M_{e}^{*}$, violating Lemma 6.1.

Lemma 6.3. For $G=(V ; E)$ and $H=(W ; F) \in \mathbf{G}_{\mathbf{c}}$, if $h: G \longrightarrow H$ is a compatible mapping, then $\mathbf{\Phi}(h)\left(S_{G}\right) \not S_{H}^{*}$.

Proof. Let $(A, B)_{e} \in M_{e}$. By Lemma 6.2, $\bar{\pi}_{G}\left((A, B)_{e}\right) \notin S_{G}^{*}$ iff $(A, B)_{e} \notin M_{e}^{*}$ which is the case iff $A \neq \emptyset$ and $B \subset \mathrm{V}$, by the properties of $M_{e}$. Since $\boldsymbol{\Phi}(h)\left(\bar{\pi}_{G}\left((A, B)_{e}\right)\right)=$ $\bar{\pi}_{H}\left((A, B)_{(h(u), h(v))}\right)$ for $e=(u, v) \in E, \boldsymbol{\Phi}(h)\left(S_{G}\right) \nsubseteq S_{H}^{*}$.

Thus Lemma 6.3 provides part (i) of our proof of Theorem 1.1.

The following applies to any pseudocomplemented semilattice $S$. Let $Z$ be any generating set for $S$, and $x \in S$. Then $x$ may be written as $x=x^{* *} \wedge z_{0} \wedge \cdots \wedge z_{n-1}$ for some $n \in \mathbb{N}$ and $z_{i} \in Z \backslash S^{*}$ (see, for example, [29]). In any such representation, $x \notin S^{*}$ iff $n \neq 0$; thus, $x \notin S^{*}$ implies $x \leq z$ for some $z \in Z \backslash S^{*}$.

Lemma 6.4. For $y, z \in S_{G}$, if $0 \neq y^{*} \leq z$, then $z \in S_{G}^{*}$.

Proof. By Lemma 2.3, $S_{G}$ is generated by $\left\{\bar{\pi}_{G}\left((\mathrm{~V} \backslash\{\mathrm{x}\}, \mathrm{V} \backslash\{\mathrm{x}\})_{e}\right): \mathrm{x} \in \mathrm{V}\right.$ and $e \in$ $E\}$. Assume $y, z \in S_{G}$ and $y^{*} \leq z \notin S_{G}^{*}$. Thus $y^{*} \leq \bar{\pi}_{G}\left((\vee \backslash\{\mathrm{x}\}, \mathrm{V} \backslash\{\mathrm{x}\})_{e}\right)$ for some $\mathrm{x} \in \mathrm{V}$ and $e \in E$ by the preceding remark. Now $\varphi_{G}\left(y^{*}\right) \in B_{G} \llbracket F_{G} \rrbracket^{*}$ while $\varphi_{G}\left(\bar{\pi}_{G}\left((\mathrm{~V} \backslash\{\mathrm{x}\}, \mathrm{V} \backslash\{\mathrm{x}\})_{e}\right)\right)=\bar{\varphi}\left((\mathrm{V} \backslash\{\mathrm{x}\}, \mathrm{V} \backslash\{\mathrm{x}\})_{e}\right)=\varphi_{e}\left((\mathrm{~V} \backslash\{\mathrm{x}\}, \mathrm{V} \backslash\{\mathrm{x}\})_{e}\right)=$ $\left(\pi_{G}\left(\left(\mathrm{~V}_{e} \backslash\left\{\mathrm{x}_{e}\right\}\right)^{\prime}\right), \emptyset\right) \notin B_{G} \llbracket F_{G} \rrbracket^{*}$. As $\varphi_{G}$ is order-preserving, this implies $\varphi_{G}\left(y^{*}\right)=$ $(\emptyset, \emptyset)=0$ in $B_{G} \llbracket F_{G} \rrbracket^{*}$ and thus $y^{*}=0$ since $\varphi_{G}$ is one-to-one on $S_{G}^{*} \cong B_{G} \llbracket F_{G} \rrbracket^{*} \cong$ $B_{G}$.

Lemma 6.5. For $\mathrm{x} \in \mathrm{V}$ and $e \in E$, the Glivenko class of $\bar{\pi}_{G}\left((\mathrm{~V} \backslash\{\mathrm{x}\}, \mathrm{V})_{e}\right)$ in $S_{G}$ has precisely two elements, namely $\bar{\pi}_{G}\left((\mathrm{~V} \backslash\{\mathrm{x}\}, \mathrm{V})_{e}\right)$ and $\bar{\pi}_{G}\left((\mathrm{~V} \backslash\{\mathrm{x}\}, \mathrm{V} \backslash\{\mathrm{x}\})_{e}\right)$.

Proof. By definition, the Glivenko class of $(\mathrm{V} \backslash\{\mathrm{x}\}, \mathrm{V})_{e}$ in $M_{e}$ has precisely two elements, namely $(\mathrm{V} \backslash\{\mathrm{x}\}, \mathrm{V})_{e}$ and $(\mathrm{V} \backslash\{\mathrm{x}\}, \mathrm{V} \backslash\{\mathrm{x}\})_{e}$. By Lemma 6.1, the Glivenko class of $\bar{\pi}_{G}\left((\mathrm{~V} \backslash\{\mathrm{x}\}, \mathrm{V})_{e}\right)$ in $S_{G}$ thus contains at least the two elements specified 
by the Lemma. Assume $y \in S_{G}$ is another member of this class, that is, $y^{* *}=$ $\bar{\pi}_{G}\left((\mathrm{~V} \backslash\{\mathrm{x}\}, \mathrm{V})_{e}\right)$. By the remark preceding Lemma $6.4, y$ may be written as $y=$ $y^{* *} \wedge z_{0} \wedge \cdots \wedge z_{n-1}$ where the $z_{i}$ belong to $Z \backslash S_{G}^{*}, Z$ a set generating $S_{G}$; thus $y^{* *}=$ $y^{* *} \wedge z_{0}^{* *} \wedge \cdots \wedge z_{n-1}^{* *}$ and $y^{* *} \leq z_{i}^{* *}$ for all $0 \leq i \leq n-1$. By Lemma 2.3, $Z$ may be chosen as $\left\{\bar{\pi}_{G}\left((\mathrm{~V} \backslash\{\mathrm{x}\}, \mathrm{V} \backslash\{\mathrm{x}\})_{e}\right): \mathrm{x} \in \mathrm{V}\right.$ and $\left.e \in E\right\}$, whence $Z \cap S_{G}^{*}=\emptyset$. Consider a fixed $z_{i}$. Then $z_{i}^{* *}$ has the form $\bar{\pi}_{G}\left((\mathrm{~V} \backslash\{\mathrm{w}\}, \mathrm{V})_{f}\right)=\bar{\pi}_{G}\left(\left(\mathrm{~V}_{f} \backslash\left\{\mathrm{w}_{f}\right\}, \mathrm{V}_{f}\right)\right.$ for some $f \in E$ and $\mathrm{w}_{f} \in \mathrm{\vee}_{f}$, and we obtain $y^{* *}=\bar{\pi}_{G}\left(\left(\mathrm{~V}_{e} \backslash\left\{\mathrm{x}_{e}\right\}, \mathrm{\vee}_{e}\right)\right) \leq \bar{\pi}_{G}\left(\left(\mathrm{\vee}_{f} \backslash\left\{\mathrm{w}_{f}\right\}, \mathrm{\vee}_{f}\right)\right.$. This comparability is within the Boolean algebra $B_{G}$, so we may take complements and obtain $\bar{\pi}_{G}\left(\left(\left\{\mathrm{x}_{e}\right\}, \mathrm{V}_{e}\right)\right) \geq \bar{\pi}_{G}\left(\left(\left\{\mathrm{w}_{f}\right\}, \mathrm{V}_{f}\right)\right.$, in terms of $B_{G}$ as constructed in $\S 4$ this reads as $\pi_{G}\left(\mathrm{x}_{e}^{\prime}\right) \geq \pi_{G}\left(\mathrm{w}_{f}^{\prime}\right)$. By Lemma 4.5 we obtain $\pi_{G}\left(\mathrm{x}_{e}^{\prime}\right)=\pi_{G}\left(\mathrm{w}_{f}^{\prime}\right)$, thus back in terms of $S_{G}$ we have $\bar{\pi}_{G}\left((\mathrm{~V} \backslash\{\mathrm{x}\}, \mathrm{V})_{e}\right)=\bar{\pi}_{G}\left((\mathrm{~V} \backslash\{\mathrm{w}\}, \mathrm{V})_{f}\right)$. By the definition of $\Theta_{G}$ then also $\bar{\pi}_{G}\left((\mathrm{~V} \backslash\{\mathrm{x}\}, \mathrm{V} \backslash\{\mathrm{x}\})_{e}\right)=\bar{\pi}_{G}\left((\mathrm{~V} \backslash\{\mathrm{w}\}, \mathrm{V} \backslash\{\mathrm{w}\})_{f}\right)$ and we are done since this applies for every $z_{i}(0 \leq i \leq n-1)$.

To obtain part (ii) of the proof of Theorem 1.1, assume that, for $G=(V, E)$ and $H=(W, F) \in \mathbf{G}_{\mathbf{c}}, \varphi: S_{G} \longrightarrow S_{H}$ is any homomorphism for which $\varphi\left(S_{G}\right) \nsubseteq S_{H}^{*}$. It must be shown that $\varphi=\Phi(h)$ for some compatible map $h: G \longrightarrow H$.

Suppose that, for some $\mathrm{x} \in \mathrm{V}$ and $e \in E, \varphi\left(\bar{\pi}_{G}\left((\mathrm{~V} \backslash\{\mathrm{x}\}, \mathrm{V})_{e}\right)\right)=\varphi\left(\bar{\pi}_{G}((\mathrm{~V} \backslash\right.$ $\left.\{\mathrm{x}\}, \mathrm{V} \backslash\{\mathrm{x}\})_{e}\right)$ ). Denoting the restriction of $\varphi \circ \bar{\pi}_{G}$ to $M_{e} \subseteq S$ by $\alpha$, we obtain by Lemma 2.5 that either ker $\alpha \supseteq \Gamma_{M_{e}}$ or else that there exist $(A, B)_{e},(C, D)_{e} \in M_{e}$ such that $\alpha\left((A, B)_{e}\right) \neq \alpha\left((A, \mathrm{~V})_{e}\right)$ and $\alpha\left((A, B)_{e}\right) \geq \alpha\left((C, D)_{e}^{*}\right) \neq \alpha\left((\emptyset, \emptyset)_{e}\right)$. The latter option is ruled out by Lemma 6.4, thus ker $\alpha \supseteq \Gamma_{M_{e}}$. In particular, we have $\left.\alpha(\mathrm{V} \backslash\{\mathrm{x}\}, \mathrm{V})_{e}\right)=\alpha\left((\mathrm{V} \backslash\{\mathrm{x}\}, \mathrm{V} \backslash\{\mathrm{x}\})_{e}\right)$ for all $\mathrm{x} \in \mathrm{V}$. Consider $f \in E$ such that either $\sigma f=\tau e$ or $\tau f=\sigma e$. Then, by the definition of $\Theta_{G}$, we have $\bar{\pi}_{G}((\mathrm{~V} \backslash\{\mathrm{a}\}, \mathrm{V} \backslash$ $\left.\{\mathrm{a}\})_{e}\right)=\bar{\pi}_{G}\left((\mathrm{~V} \backslash\{\mathrm{b}\}, \mathrm{V} \backslash\{\mathrm{b}\})_{f}\right)$ or $\left.\bar{\pi}_{G}\left((\mathrm{~V} \backslash\{\mathrm{a}\}, \mathrm{V} \backslash\{\mathrm{a}\})_{f}\right)\right)=\bar{\pi}_{G}\left((\mathrm{~V} \backslash\{\mathrm{b}\}, \mathrm{V} \backslash\{\mathrm{b}\})_{e}\right)$, hence $\bar{\pi}_{G}\left((\mathrm{~V} \backslash\{\mathrm{b}\}, \mathrm{V})_{f}\right)=\bar{\pi}_{G}\left((\mathrm{~V} \backslash\{\mathrm{b}\}, \mathrm{V} \backslash\{\mathrm{b}\})_{f}\right)$ or $\bar{\pi}_{G}\left((\mathrm{~V} \backslash\{\mathrm{a}\}, \mathrm{V})_{f}\right)=\bar{\pi}_{G}((\mathrm{~V} \backslash$ $\left.\{\mathrm{a}\}, \mathrm{V} \backslash\{\mathrm{a}\})_{f}\right)$. So there is some $\mathrm{y} \in \mathrm{V}$ and such that $\varphi\left(\bar{\pi}_{G}\left((\mathrm{~V} \backslash\{\mathrm{y}\}, \mathrm{V})_{f}\right)\right)=$ $\varphi\left(\bar{\pi}_{G}\left((\mathrm{~V} \backslash\{\mathrm{y}\}, \mathrm{V} \backslash\{\mathrm{y}\})_{f}\right)\right)$. Repeating the procedure and using connectivity of $G$, this implies by Lemma 6.5 that $\varphi$ collapses the full Glivenko classes of all generators of $S_{G}$, implying $\varphi\left(S_{G}\right) \subseteq S_{H}^{*}$, contrary to hypothesis.

We conclude that $\varphi\left(\bar{\pi}_{G}\left((\mathrm{~V} \backslash\{\mathrm{x}\}, \mathrm{V})_{e}\right)\right) \neq \varphi\left(\bar{\pi}_{G}\left((\mathrm{~V} \backslash\{\mathrm{x}\}, \mathrm{V} \backslash\{\mathrm{x}\})_{e}\right)\right)$ for every $\mathrm{x} \in \mathrm{V}$ and every $e \in E$.

Observe that $S_{H}$ is generated by $\left\{\bar{\pi}_{H}\left((\mathrm{~V} \backslash\{\mathrm{y}\}, \mathrm{V} \backslash\{\mathrm{y}\})_{f}\right): \mathrm{y} \in \mathrm{V}\right.$ and $\left.f \in F\right\}$. Since, for every $\mathrm{x} \in \mathrm{V}$ and every $e \in E, \varphi\left(\bar{\pi}_{G}\left((\mathrm{~V} \backslash\{\mathrm{x}\}, \mathrm{V})_{e}\right)\right) \neq \varphi\left(\bar{\pi}_{G}((\mathrm{~V} \backslash\{\mathrm{x}\}, \mathrm{V} \backslash\right.$ $\left.\left.\{\mathrm{x}\})_{e}\right)\right)$, we conclude that, for each $\mathrm{x} \in \mathrm{V}$ and $e \in E, \varphi\left(\bar{\pi}_{G}\left((\mathrm{~V} \backslash\{\mathrm{x}\}, \mathrm{V})_{e}\right)\right) \leq$ $\bar{\pi}_{H}\left((\mathrm{~V} \backslash\{\mathrm{y}\}, \mathrm{V})_{f}\right)$ for some $\mathrm{y} \in \mathrm{V}$ and $f \in F$. Separated as it is by $\S 5$, Corollary 4.12 feels a long way away. However, this is precisely where it is needed. For each $e \in E$, by Corollary 4.12 with $\left\{y_{i}: 1 \leq i \leq 6\right\}=\phi\left(\bar{\pi}_{G}\left((\mathrm{~V} \backslash\{\mathrm{x}\}, \mathrm{V})_{e}\right): \mathrm{x} \in \mathrm{V}\right\}$, there exists $\eta(e) \in F$ such that $\phi\left(\bar{\pi}_{G}\left((\mathrm{~V} \backslash\{\mathrm{x}\}, \mathrm{V})_{e}\right)=\bar{\pi}_{H}\left((\mathrm{~V} \backslash\{\zeta(\mathrm{x})\}, \mathrm{V})_{\eta(e)}\right)\right.$ where $\zeta: \mathrm{V} \longrightarrow \mathrm{V}$ is one-to-one (and thus bijective). Furthermore, by Lemma $6.5, \varphi\left(\bar{\pi}_{G}((\mathrm{~V} \backslash\{\mathrm{x}\}, \mathrm{V} \backslash\right.$ 
$\left.\left.\{\mathrm{x}\})_{e}\right)\right)=\bar{\pi}_{H}\left((\mathrm{~V} \backslash\{\zeta(\mathrm{x})\}, \mathrm{V} \backslash\{\zeta(\mathrm{x})\})_{\eta(e)}\right)$. By Lemma 2.7, $\zeta$ is the identity, that is, for every $\mathrm{x} \in \mathrm{V}$ and $e \in E, \varphi\left(\bar{\pi}_{G}\left((\mathrm{~V} \backslash\{\mathrm{x}\}, \mathrm{V})_{e}\right)\right)=\bar{\pi}_{H}\left((\mathrm{~V} \backslash\{\mathrm{x}\}, \mathrm{V})_{\eta(e)}\right)$ and $\varphi\left(\bar{\pi}_{G}\left((\mathrm{~V} \backslash\{\mathrm{x}\}, \mathrm{V} \backslash\{\mathrm{x}\})_{e}\right)\right)=\bar{\pi}_{H}\left((\mathrm{~V} \backslash\{\mathrm{x}\}, \mathrm{V} \backslash\{\mathrm{x}\})_{\eta(e)}\right)$.

Consider $v \in V$. Then, since $G \in \mathbf{G}_{\mathbf{c}}$, there exist $e_{1}, e_{2} \in E$ such that $\tau e_{1}=$ $v=\sigma e_{2}$. In particular, $\bar{\pi}_{G}\left((\mathrm{~V} \backslash\{\mathrm{a}\}, \mathrm{V})_{e_{1}}\right)=\bar{\pi}_{G}\left((\mathrm{~V} \backslash\{\mathrm{b}\}, \mathrm{V})_{e_{2}}\right)$. Thus, $\varphi\left(\bar{\pi}_{G}((\mathrm{~V} \backslash\right.$ $\left.\left.\{\mathrm{a}\}, \mathrm{V})_{e_{1}}\right)\right)=\varphi\left(\bar{\pi}_{G}\left((\mathrm{~V} \backslash\{\mathrm{b}\}, \mathrm{V})_{e_{2}}\right)\right)$. Whence, $\varphi\left(\bar{\pi}_{G}\left((\mathrm{~V} \backslash\{\mathrm{a}\}, \mathrm{V})_{\eta\left(e_{1}\right)}\right)\right)=\varphi\left(\bar{\pi}_{G}((\mathrm{~V} \backslash\right.$ $\left.\left.\{\mathrm{b}\}, \mathrm{V})_{\eta\left(e_{2}\right)}\right)\right)$. Even further away are Corollary 4.6 and Lemma 4.7. However, this is the point where they are needed. By Corollary 4.6 and Lemma 4.7, this is only possible if $\eta\left(e_{1}\right)=f_{1}$ and $\eta\left(e_{2}\right)=f_{2}$, for $f_{1}, f_{2} \in F$ such that $\tau f_{1}=w=\sigma f_{2}$. Set $h(v)=w$. By Corollary 4.6 and Lemma 4.7, $h: V \longrightarrow W$ is a well-defined compatible mapping. Since $\boldsymbol{\Phi}(h)\left(\bar{\pi}_{G}\left((V \backslash\{\mathrm{x}\}, V \backslash\{\mathrm{x}\})_{e}\right)\right)=\varphi\left(\bar{\pi}_{G}((V \backslash\{\mathrm{x}\}, V \backslash\right.$ $\left.\left.\{\mathrm{x}\})_{e}\right)\right)$ for every $\mathrm{x} \in \mathrm{V}$ and $e \in E$ and $S_{G}$ is generated by $\left\{(\mathrm{V} \backslash\{\mathrm{x}\}, \mathrm{V} \backslash\{\mathrm{x}\})_{e}: \mathrm{x} \in\right.$ $\vee$ and $e \in E$, we have $\varphi=\boldsymbol{\Phi}(h)$, as required.

\section{Concluding remark}

A pseudocomplemented semilattice is relatively rigid providing the only endomorphism $\varphi: S \longrightarrow S$ for which $\varphi(S) \nsubseteq S^{*}$ is the identity. Since there exists a proper class of non-isomorphic rigid connected strongly loopless graphs, it follows from Theorem 1.1 that there exists a proper class of non-isomorphic relatively rigid pseudocomplemented semilattices. Inspection of the functor $\boldsymbol{\Phi}$ shows that, for a graph $G=(V ; E) \in \mathbf{G}_{\mathbf{c}},\left|\Phi\left(S_{G}\right)^{*}\right| \geq|E|$, which increases with $|V|$. This leads us to the following problem. Does there exist some cardinal $\kappa$ for which there is a proper class of non-isomorphic relatively rigid pseudocomplemented semilattices such that, for each member $S,\left|S^{*}\right| \leq \kappa$ ?

Since the time of submission, Václav Koubek and Jiří Sichler have shown that every finite-to-finite almost universal variety $\mathbf{V}$ is $Q$-universal (Almost ff-universality imples $Q$-universality, to appear).

Acknowledgement. We would like to express our thanks to the referee, whose thoughtful comments greatly improved the presentation of this paper.

\section{REFERENCES}

[1] M. E. Adams and W. Dziobiak, Finite-to-finite universal quasivarieties are Q-universal, Algebra Universalis 46 (2001), 253-283.

[2] M. E. Adams, W. Dziobiak, M. Gould, and J. Schmid, Quasivarieties of pseudocomplemented semilattices, Fund. Math. 146 (1995), 295-312.

[3] M. E. Adams and M. Gould, A construction for pseudocomplemented semilattices and two applications, Proc. Amer. Math. Soc. 106 (1989), 899-905. 
[4] R. Balbes, On free pseudo-complemented and relatively pseudo-complemented semilattices, Fund. Math. 78 (1973), 119-131.

[5] G. Birkhoff, On the structure of abstract algebras, Proc. Cambridge Philos. Soc. 31 (1935), 433-454.

[6] M. Demlová and V. Koubek, Endomorphism monoids of bands, Semigroup Forum 38 (1989), 305-329.

[7] M. Demlová and V. Koubek, Endomorphism monoids in small varieties of bands, Acta Sci. Math. (Szeged) 55 (1991), 9-20.

[8] M. Demlová and V. Koubek, Endomorphism monoids in varieties of bands, Acta Sci. Math. (Szeged) 66 (2000), 477-516.

[9] O. Frink, Pseudo-complements in semilattices, Duke Math. J. 29 (1962), 505-514.

[10] V. A. Gorbunov, Algebraic Theory of Quasivarieties, Plenum Publishing Co., New York, 1998.

[11] G. Grätzer and J. Sichler, On the endomorphism semigroup (and category) of bounded lattices, Pacific J. Math. 35 (1970), 639-647.

[12] Z. Hedrlín and A. Pultr, Symmetric relations (undirected graphs) with given semigroup, Monatsh. Math. 69 (1965), 318-322.

[13] Z. Hedrlín and A. Pultr, On full embeddings of categories of algebras, Illinois J. Math. 10 (1966), 392-406.

[14] G. T. Jones, Pseudocomplemented semilattices, Ph.D. dissertation, U.C.L.A., 1972.

[15] G. T. Jones, Projective pseudo-complemented semilattices, Pacific J. Math. 52 (1974), 443-456.

[16] T. Katriňák and Z. Heleyová, Free products of pseudocomplemented semilattices, Semigroup Forum 60 (2000), 450-469.

[17] S. Koppelberg, Handbook of Boolean Algebras I, North-Holland, Amsterdam, 1989.

[18] V. Koubek and J. Sichler, Universal varieties of semigroups, J. Austral. Math. Soc. (Series A) 36 (1984), 143-152.

[19] V. Koubek and J. Sichler, On relative universality and Q-universality, Studia Logica 78 (2004), 279-291.

[20] K. D. Magill, The semigroup of endomorphisms of a Boolean ring, Semigroup Forum 4 (1972), 411-416.

[21] C. J. Maxson, On semigroups of Boolean ring endomorphisms, Semigroup Forum 4 (1972), $78-82$.

[22] E. Mendelsohn, On a technique for representing semigroups as endomorphism semigroups of graphs with given properties, Semigroup Forum 4 (1972), 283-294.

[23] A. Pultr, Concerning universal categories, Comment. Math. Univ. Carolinae 6 (1964), 227-239.

[24] A. Pultr and V. Trnková, Combinatorial, Algebraic, and Topological Representations of Groups, Semigroups, and Categories, North-Holland, Amsterdam, 1980.

[25] H. P. Sankappanavar, Principal congruences of pseudocomplemented semilattices and congruence extension property, Proc. Amer. Math. Soc. 73 (1979), 308-312.

[26] H. P. Sankappanavar, Remarks on subdirectly irreducible pseudocomplemented semi-lattices and distributive pseudocomplemented lattices, Math. Japon. 25 (1980), 519-521.

[27] B. M. Schein, Ordered sets, semilattices, distributive lattices, and Boolean algebras with homomorphic endomorphism semigroups, Fund. Math. 68 (1970), 31-50.

[28] J. Schmid, Lee classes and sentences for pseudocomplemented semilattices, Algebra Universalis 25 (1988), 223-232.

[29] J. Schmid, On the structure of free pseudocomplemented semilattices, Houston J. Math. 16 (1990), 71-85. 
[30] J. Sichler, Nonconstant endomorphisms of lattices, Proc. Amer. Math. Soc. 34 (1972), $67-70$.

[31] P. Vopěnka, Z. Hedrlín, and A. Pultr, A rigid relation exists on any set, Comment. Math. Univ. Carolinae 6 (1965), 149-155.

M. E. ADAMS

Department of Mathematics, State University of New York, New Paltz, NY 12561, USA

e-mail: adamsm@newpaltz.edu

\section{JÜRG SCHMID}

Institute of Mathematics, University of Bern, CH-3012 Bern, Switzerland

e-mail: juerg.schmid@math.unibe.ch 